Merci d'utiliser le titre suivant lorsque vous citez ce document :

Giner, C. (2010-01-20), « Nouvelles pistes de création de valeur dans le secteur agroalimentaire », Éditions OCDE, Paris.

http://dx.doi.org/10.1787/5kmmmx8ktfmt-fr

\title{
Nouvelles pistes de création de valeur dans le secteur agroalimentaire
}

\section{Céline Giner}

La version originale de ce document a été publiée comme suit :

Giner, C. (2009-01-01), "New Avenues of Value Creation in the Agro-Food Sector", OECD Food, Agriculture and Fisheries Papers, No. 13, OECD Publishing, Paris. http://dx.doi.org/10.1787/221300175226 
Organisation de Coopération et de Développement Économiques

Organisation for Economic Co-operation and Development

20-Jan-2009

DIRECTION DES ÉCHANGES ET DE L'AGRICULTURE

Français - Or. Anglais

COMITÉ DE L'AGRICULTURE

Groupe de travail des politiques et des marchés agricoles

NOUVELLES PISTES DE CRÉATION DE VALEUR DANS LE SECTEUR AGROALIMENTAIRE

Personne à contacter: Céline Giner (e-mail: celine.giner@oecd.org)

JT03258394

Document complet disponible sur OLIS dans son format d'origine

Complete document available on OLIS in its original format 


\section{NOTE}

Ce document est présenté dans le cadre du programme de travail 2007-2008. Il donne des pistes de réflexion sur les nouvelles sources de création et de captation de valeur au sein du secteur agroalimentaire.

Le document est illustré de cinq études de cas qui ont été préparées en collaboration avec des acteurs du secteur agro-alimentaire de différents pays de l'OCDE. L'auteur principal du rapport est Céline Giner. Linda Fulponi a pris part à la rédaction. Shingo Kimura, Mauro Migotto et Michael Stennis ont participé aux recherches nécessaires à l'élaboration de ce papier. 


\section{Table des matières}

NOUVELLES PISTES DE CRÉATION DE VALEUR DANS LE SECTEUR AGROALIMENTAIRE .... 4

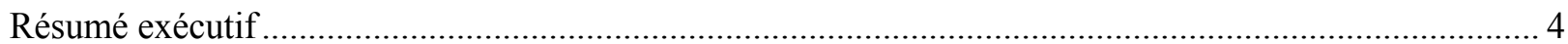

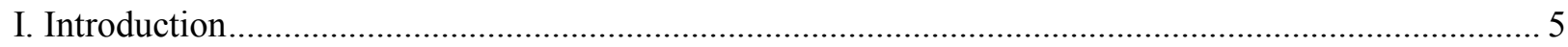

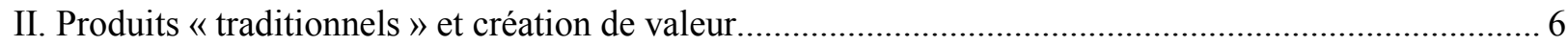

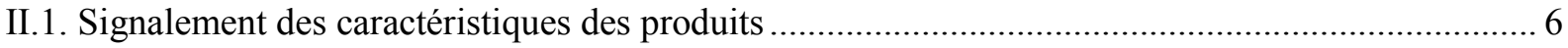

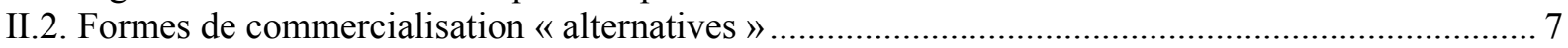

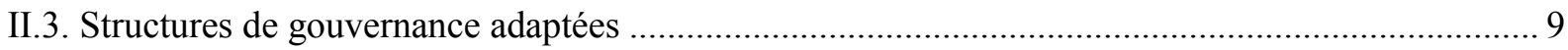

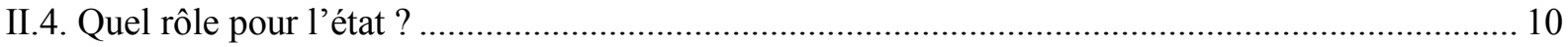

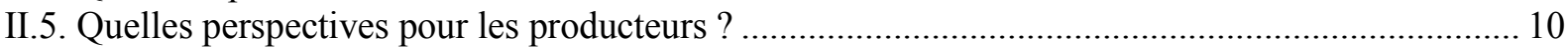

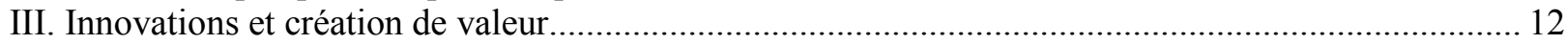

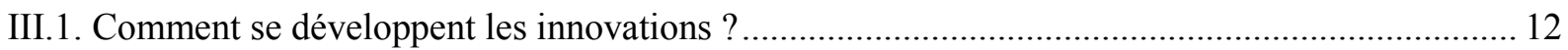

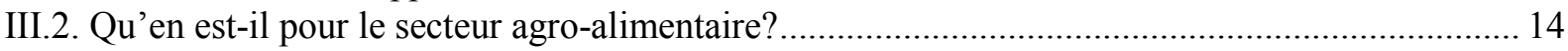

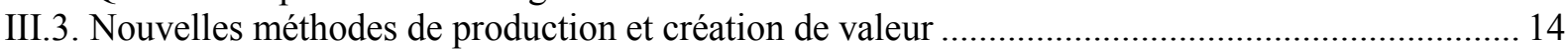

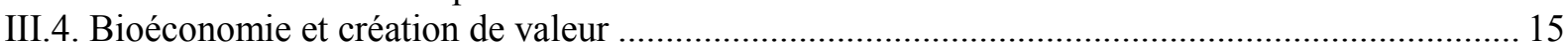

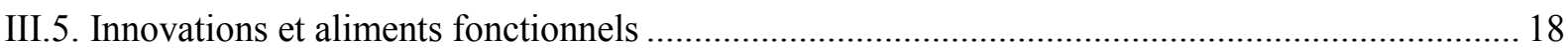

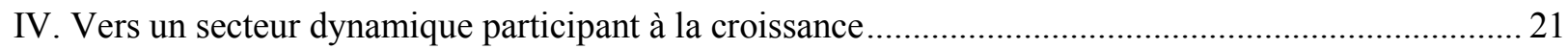

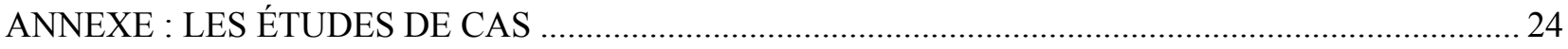

A-I. Le développement de circuits alternatifs de distribution alimentaire (Japon) ................................. 24

A-II. Irrigation au goutte à goutte-fertirrigation et techniques nucléaires (Turquie) ............................... 26

A-III. Pôle de compétitivité Industries et Agro-Ressources (France) ……….......................................... 29

A-IV. Quelques exemples d'aliments fonctionnels enrichis en oméga-3 (France, Canada, Allemagne) . 32

A-V. Des oléagineux enrichis en oméga-3 pour la durabilité et la santé (Australie) ............................... 34

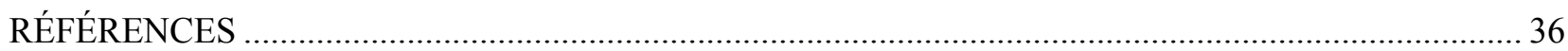




\section{NOUVELLES PISTES DE CRÉATION DE VALEUR DANS LE SECTEUR AGROALIMENTAIRE}

\section{Résumé exécutif}

1. Le document donne des pistes de réflexion sur les nouvelles sources de création et de captation de valeur au sein du secteur agro-alimentaire. L'ensemble des opportunités de création de valeur est vaste et le document n'est pas exhaustif. De nouvelles stratégies sont en cours d'élaboration par les différents acteurs du secteur dans un contexte mondialisé incertain et compétitif où les attentes des consommateurs et de la société en général évoluent.

2. La première section du rapport met l'accent sur le fait que les produits «traditionnels » peuvent être une source de création de valeur pour les acteurs du secteur agro-alimentaire. Les producteurs agricoles peuvent en particulier valoriser leur rôle au sein du secteur s'ils sont parties prenantes d'une réorganisation de la chaîne de valeur et s'ils participent à la mise en avant de certaines caractéristiques «spécifiques» des produits. Pour illustrer ce fait, une étude de cas en annexe présente l'évolution des circuits de commercialisation des fruits et légumes au Japon. Il ressort de cette première section que les transformations du secteur agro-alimentaire peuvent être facilitées si les gouvernements soutiennent la mise en place de systèmes de signalisation adaptés, s'ils permettent à des systèmes d'organisation flexibles et innovants de voir le jour ou encore s'ils encouragent la coopération entre les acteurs du secteur.

3. La seconde section traite de l'élargissement de l'ensemble des possibilités de création de valeur au sein du secteur agro-alimentaire avec le développement d'innovations qui se traduisent par la mise sur le marché de nouveaux produits et par l'adoption de nouvelles méthodes de production. Le rapport étudie comment les producteurs peuvent être associés à ce processus de création de valeur. Cette section est illustrée en annexe par quatre études de cas qui ont pour sujets le développement de nouvelles méthodes de production et le développement de nouveaux produits, en particulier des aliments fonctionnels et des bioproduits. La seconde section et les exemples associés illustrent les faits suivants:

- le secteur agro-alimentaire peut tirer partie d'innovations développées par d'autres secteurs de l'économie et/ou investir dans des activités de recherche et développement (R\&D),

- l'état peut favoriser le dynamisme du secteur en facilitant le développement d'innovations,

- l'état peut aussi aider à l'émergence de structures de coopérations dynamiques au sein de certains territoires qui permettent au secteur public et au secteur privé de collaborer.

4. Le papier conclut sur les capacités du secteur agro-alimentaire à être à l'avenir un secteur innovant capable de générer de la richesse pour l'ensemble de l'économie. 


\section{Introduction}

5. L'analyse des stratégies à disposition des producteurs pour créer et capter de la valeur au sein du secteur agro-alimentaire des pays de l'OCDE fait partie du programme de travail 2007-2008. On distingue souvent les grandes exploitations agricoles bénéficiant d'économies d'échelle des petites exploitations familiales. Dans les faits, les structures de production agricole sont hétérogènes. Le processus de création et de captation de valeur est donc différent d'un producteur à l'autre et il est difficile de définir des stratégies types permettant aux producteurs de maximiser leurs revenus à court, moyen ou long terme ou tout simplement de rester économiquement viables. Même si les prix des matières premières agricoles se maintiennent à des niveaux durablement plus élevés que par le passé, il n'en demeure pas moins probable que les producteurs des pays de l'OCDE soient soumis à un environnement incertain et compétitif. Ceci pourrait être le résultat de la combinaison d'un ensemble de facteurs comme, entre autres, la disparition progressive des politiques de subvention agricoles, la montée en puissance de la grande distribution et des multinationales agro-alimentaires, la libéralisation graduelle des échanges internationaux et la concurrence associée en provenance des pays en voie de développement.

6. Dans ce contexte, les producteurs devraient être amenés à renouveler l'ensemble des possibilités qui s'offrent à eux en matière de création et de captation de valeur. Étant donnée la complexité des connections entre les différentes activités de la chaîne agro-alimentaire, il est impossible d'appréhender le processus de création de valeur pour les producteurs indépendamment des autres acteurs de la chaîne. Le rôle des producteurs au sein du système agro-alimentaire devrait donc évoluer et, pour ce faire, des structures d'organisation innovantes et adaptées liant les producteurs aux industriels, au monde de la recherche et à la grande distribution devraient se développer plus avant.

7. L'évolution des formes de gouvernance de la chaîne agro-alimentaire est aussi une nécessité au vue de la modification des attentes des consommateurs. La société demande en effet au secteur d'assurer à la fois la sécurité alimentaire mais aussi tout un ensemble d'objectifs complémentaires en matière de santé ou de préservation des ressources et de l'environnement. Le secteur agricole doit donc proposer des produits adaptés aux besoins des consommateurs. Ces produits peuvent prendre la forme de produits «traditionnels » dont certains attributs sont mis en avant comme par exemple la qualité, le mode de production ou le système de commercialisation. La forme d'organisation choisie au sein de la chaîne déterminera alors couramment les possibilités de création de valeur pour les producteurs. L'évolution des attentes de la société en matière de santé et de préoccupations environnementales devrait aussi avoir pour conséquence le développement de produits innovants très différents des produits traditionnellement issus $\mathrm{du}$ secteur agro-alimentaire. Le recours à l'innovation devrait mener au développement de nouvelles techniques de production et à la mise sur le marché de façon soutenue de bioproduits produits fabriqués à partir de ressources renouvelables et d'aliments fonctionnels aliments et composants alimentaires susceptibles d'être plus bénéfiques à la santé que les aliments ordinaires.

8. Les bioproduits vont bien au-delà des simples produits alimentaires. Il peut s'agir de matériaux de construction comme les biomatériaux, de produits chimiques dits "verts » ou encore de carburants issus de la biomasse comme les biocarburants. Le développement des bioproduits nécessitera des investissements spécifiques en matière de recherche et développement pour permettre à des innovations de voir le jour. Ces innovations devraient être rendues possibles par la diminution des coûts liés aux nouvelles technologies et par la collaboration entre de nombreux acteurs allant du producteur à l'état, en passant par des entreprises privées de recherche et développement ou des industries spécifiques.

9. L'objectif principal du rapport est de donner aux délégations des pistes de réflexion sur les nouvelles opportunités de création et de captation de valeur amenées à se développer au sein du secteur agro-alimentaire dans un contexte mondialisé incertain et compétitif. Le rapport ne prétend en aucun cas être exhaustif. L'ensemble des possibilités est vaste et la solution choisie par un producteur pour 
maximiser ses revenus dépendra de ses spécificités. Cependant ce choix sera le résultat d'une combinaison entre positionnement du producteur au sein de la chaîne agro-alimentaire et orientation de la production en réponse à la demande des consommateurs.

10. La première section du rapport cherchera à comprendre comment le secteur agro-alimentaire et les producteurs en particulier peuvent répondre de façon adaptée à l'évolution des attentes des consommateurs en misant sur les produits «traditionnels». La mise en avant de caractéristiques spécifiques et la réorganisation de la chaîne agro-alimentaire pourraient permettre aux producteurs de valoriser leur rôle au sein du secteur.

11. La seconde section du rapport discutera de la manière dont le secteur agro-alimentaire peut élargir son ensemble de possibilités en misant sur les innovations associées entre autres au développement de nouveaux produits ou à l'utilisation de nouvelles méthodes de production agricole. Le potentiel pour les producteurs de tirer partie de ces innovations sera analysé en soulignant les besoins en structures d'organisation adaptées prenant souvent la forme de réseaux reliant les différents acteurs du secteur.

12. Un des objectifs du rapport est de comprendre dans quelle mesure le secteur agro-alimentaire pourrait à l'avenir être un secteur innovant capable de générer de la richesse pour l'ensemble de l'économie. Dans ce contexte, il sera mis en avant que l'état peut avoir un rôle de facilitateur du processus d'innovation et des orientations choisies par les producteurs et le secteur agro-alimentaire dans son ensemble.

\section{Produits « traditionnels » et création de valeur}

13. Les attentes des consommateurs envers leur alimentation sont diverses. Une chose est cependant certaine, les consommateurs veulent avoir accès à une nourriture sûre d'un point de vue sanitaire ${ }^{1}$. Ensuite, des facteurs comme le prix, la praticité, le goût, la qualité, le mode de fabrication et de commercialisation, les apports nutritionnels et l'origine des produits entrent en ligne de compte (Blandford, 2000).

\section{II.1. Signalement des caractéristiques des produits}

14. Signaler certaines caractéristiques d'un produit comme par exemple l'origine géographique, la qualité, le mode de production, le mode de commercialisation ou le mode de transformation - attributs pour lesquels certains consommateurs sont prêts à payer un prix plus élevé - est l'illustration d'une stratégie de différenciation. La différenciation des produits et la création de valeur associée sont au cœur des théories générales du marketing (Lindgreen and Wynstra, 2005). L'objectif de la différenciation est de présenter sur le marché une offre similaire à celle disponible couramment, mais difficilement comparable à celle des concurrents.

15. Dans le secteur agro-alimentaire, les consommateurs ne sont pas souvent en mesure de connaître la véracité des informations sur les caractéristiques des produits. Il existe une certaine asymétrie d'information. Pour maintenir la confiance des consommateurs, la signalisation des caractéristiques prend souvent la forme d'un label, c'est-à-dire d'un attribut qui signale des caractéristiques non visibles à l'achat. Ces caractéristiques - qui répondent souvent à un cahier des charges très précis - sont contrôlées durant le cycle de production et de commercialisation par un organisme officiel indépendant qui garantie la fiabilité

1 Les résultats de l'enquête auprès des organisations de consommateurs rapportés dans le rapport [AGR/CA/APM(2004)22] «L'évolution des habitudes alimentaires » ont montré que les consommateurs attendent des gouvernements qu'ils répondent à leurs préoccupations concernant des problèmes spécifiques tels que la sécurité alimentaire (70\%) et l'étiquetage des aliments $(65 \%)$. 
et la véracité du label. De tels signaux sont susceptibles de générer de la valeur mesurée notamment par la différence des dispositions à payer des consommateurs entre un produit labellisé et un produit de référence (Loureiro et McCluskey, 2000).

16. Les labels de qualité ou d'indication d'origine protégée font depuis longtemps partie des caractéristiques prises en compte par les consommateurs au moment de l'achat des produits alimentaires (OCDE, 2000). Les stratégies de différenciation des produits alimentaires qui se basent sur ces labels ont été présentées dans le papier "Création et captation de valeur dans les filières agro-alimentaires » [TAD/CA/APM/WP(2007)16]. Ce papier souligne l'importance des formes d'organisation associées à la signalisation des spécificités du produit sur le processus de création de valeur. Les producteurs tireront mieux partie du signal de qualité s'ils le contrôlent, que ce soit à titre individuel ou par l'intermédiaire d'un collectif de producteurs.

17. Les nouvelles attentes des consommateurs notamment en matière de santé et de préservation de l'environnement donnent lieu au développement de nouveaux types de labels concernant les produits alimentaires. Ces labels peuvent fournir des informations sur le caractère biologique du produit, l'impact nutritionnel, les méthodes de production, le respect de l'environnement ou l'utilisation d'organismes génétiquement modifiés. Après une étude empirique sur ces nouveaux labels alimentaires, McCluskey et Loureiro (2003) notent que les consommateurs intéressés n'achèteront effectivement les produits présentant ces nouveaux labels avec un premium sur les produits traditionnels que s'ils les perçoivent comme étant de bonne qualité. De nouvelles opportunités de développement de niches dans le secteur agro-alimentaire sont créées par l'évolution des préoccupations de la société. Le prix et la qualité restent cependant des éléments déterminants dans les choix de consommation.

\section{II.2. Formes de commercialisation " alternatives"}

18. Les consommateurs sont souvent les moteurs du développement de nouvelles tendances agroalimentaires (Linnemann et al, 2006) et peuvent parfois même en être les initiateurs (Winter, 2003). Les préoccupations des consommateurs en matière de préservation de l'environnement couplées à celles concernant la santé peuvent pousser certains consommateurs à consommer " différemment 》 (Toyne et al, 2004 ; DuPuis, 2006 ; DuPuis and Goodman, 2005).

19. Consommer différemment peut prendre plusieurs formes : acheter des produits biologiques, peu transformés, issus du commerce équitable ou bien diminuer la distance entre lieu de production et lieu de consommation. Les chaînes de valeur qui découlent de ce nouveau mouvement de consommation ne peuvent pas encore être considérées comme les compétitrices des chaînes de valeur dominantes dans le secteur agro-alimentaire mais elles présentent certaines caractéristiques innovantes en matière de production, de mode de commercialisation et d'organisation..

20. Une des caractéristiques communes des chaînes de valeur alternatives est qu'elles rapprochent la fonction de production de la fonction de consommation. On parle de filières courtes: il y a moins d'intermédiaires. La vente des produits se fait de manière plus directe et ceci permet aux producteurs participants - souvent de petits ou moyens producteurs - d'améliorer leurs revenus.

21. Les marchés de producteurs sont un exemple de forme de commercialisation " alternative ». Aux États-Unis, le nombre de marchés de producteurs y est passé de 1785 en 1994 à 4500 en 2007 et les ventes rapportent 1 milliard de dollars par an. Les marchés de producteurs permettent aux consommateurs d'avoir accès à des produits frais. L'organisation des ces marchés de producteurs demande une forte infrastructure. La National Farmers Market Coalition ${ }^{2}$ s'est formée en 2002 grâce à l'appui de 1'USDA Agricultural

$2 \quad$ http://www.farmersmarketcoalition.org/ . 
Marketing Service et de la North American Farm Direct Marketing Association. Elle a pour but de favoriser l'échange d'information et de bonnes pratiques pour aider au développement des marchés de producteur. Les opportunités de création de valeurs engendrées par cette forme de commercialisation directe ne sont pas sans contrainte pour les producteurs impliqués. Selon Lawson et al. (2008) qui ont étudié le développement des marchés de producteurs en Nouvelle Zélande, une des nécessités à la survie des marchés de producteurs est une forte coopération entre les vendeurs.

22. Une autre possibilité de commercialisation «alternative» de produits agro-alimentaires est la vente directe de paniers de produits agricoles. Elle nécessite rarement la coopération entre plusieurs producteurs. Le producteur est le fournisseur d'un ou plusieurs groupes de consommateurs. Ceux-ci s'engagent à acheter une quote-part de la production de la saison à un prix déterminé à l'avance qui sera livrée sous la forme de paniers hebdomadaires pendant la période de récoltes. Les consommateurs prennent donc en charge une partie des risques liés aux aléas climatiques. Les choix de production sont pris par l'exploitant en accord avec le groupe de consommateurs. Ces formes de vente requièrent une forte organisation entre l'agriculteur et le noyau dirigeant des groupes de consommateurs ; celle-ci étant basée sur une confiance mutuelle.

23. Ce concept de commercialisation de produits frais est né au Japon dans les années 60 (voir l'étude de cas A-I. en annexe qui traite du développement des circuits alternatifs de distribution alimentaire au Japon ), se développe dans les pays anglo-saxons depuis les années 80 et dans certains pays européens depuis le début des années 2000. Il concerne particulièrement les fruits et légumes mais aussi les produits laitiers ou les viandes. La part des produits transformés est relativement faible. En 2007, selon Alliance PEC, un des réseaux coordinateurs français, près de 900 exploitations et 100000 consommateurs étaient concernées par ces formes de commercialisation en France $^{3}$ et 1700 exploitations et 500000 consommateurs aux États-Unis.

24. Comme pour la vente sur les marchés de producteurs, la vente collective de paniers est encore une forme anecdotique de commercialisation de produits agricoles potentiellement rémunératrice pour les producteurs quoique très intensive en temps de travail, le producteur étant en charge de la commercialisation du produit. Elle est contraignante pour les consommateurs qui ne peuvent faire tous leurs achats alimentaires sur un même lieu mais semble en adéquation avec les considérations civiques de consommation qui se développent et donc promise à un certain avenir.

25. Avec les circuits de commercialisation alternatifs décrits ci-dessus, les producteurs ont la possibilité d'engranger la majorité des bénéfices économiques de la commercialisation de leurs produits. L'absence d'intermédiaires et la relation basée sur la confiance entre producteurs et consommateurs permettent aux producteurs d'espérer des marges plus importantes. Quoique marginales au niveau global, ces pratiques alternatives sont en plein essor. Ainsi par exemple, au Japon, du fait de la diversification de la demande des consommateurs, des circuits alternatifs de commercialisation des produits alimentaires se sont développés de manière importante depuis quelques décennies. Les grossistes, qui distribuaient $82 \%$ des fruits et légumes frais en 1990, n'en distribuaient ainsi plus que $65 \%$ en 2005 . Ces circuits de commercialisation alternatifs au Japon sont l'objet de l'étude de cas A-I. présentée en annexe du rapport.

26. Il est cependant très difficile de prévoir le futur des formes de commercialisation « alternatives ». Des questions restent en suspens. Comment une relation de confiance entre producteurs et consommateurs peut-elle fonctionner à grande échelle ? Des dérives sont-elles possibles étant donnée l'asymétrie d'information entre consommateurs et producteurs? Existe-il un besoin de contrôle de ces circuits par l'état ou des organismes officiels indépendants?

3 Les consommateurs sont principalement basés en région Provence Alpes Côte d'Azur et dans les agglomérations des grandes villes. 


\section{II.3. Structures de gouvernance adaptées}

27. Le premium associé à des produits présentant des attributs spécifiques pour lesquels les consommateurs sont prêts à payer tend à diminuer lorsque la production se développe. Ainsi, les producteurs - pour maintenir leurs marges - cherchent souvent à construire des structures de gouvernance solides leur permettant de tirer au maximum partie de la signalisation des caractéristiques particulières de leurs produits.

28. Les stratégies de différenciation basées sur la signalisation de certaines caractéristiques des produits où les producteurs sont organisés de manière collective font partie de celles qui rencontrent le plus grand succès. L'Encadré 4 du papier [TAD/CA/APM/WP(2007)16] a présenté le cas de la marque de tomates Savéol qui a été développée par des coopératives de producteurs français de tomates. Savéol est une des expressions de «l'esprit d'entreprise collectif» («collective entrepreneurship ») défini par Cook et Plunkett (2006) comme un phénomène émergent dans les organisations dirigées par les producteurs. Cet « esprit d'entreprise » vise à capter des rentes à différents niveaux en misant sur l'innovation, sur la recherche de nouvelles opportunités et sur une capacité à appréhender les risques.

29. Les coopératives «traditionnelles» dont l'objectif principal est de permettre des économies d'échelle ne sont pas compétitives sur les marchés des produits novateurs par rapport à des entreprises plus flexibles (Hanf et Pieniadz, 2007). Pour pallier à ces problèmes, les coopératives de nouvelle génération ${ }^{4}$ (NGC) sont apparues depuis une vingtaine d'années (Waner, 2000). Les producteurs sont parties prenantes de la coopérative. Ils partagent les risques et les gains et ont une certaine incitation à ce que le système soit dynamique et compétitif (Chaddad et Cook, 2004). Les nouvelles formes prises par les organisations de producteurs se heurtent parfois aux systèmes légaux en place qui ne sont pas adaptés à ces entités «hybrides ${ }^{5} »$. Ces organisations ont en effet besoin de développer non seulement de nouvelles formes de propriété financières et intellectuelles mais aussi des structures séparant et limitant les risques pour les différentes parties.

30. Des structures de gouvernance adaptées permettant à une entreprise de garder à la fois le contrôle de sa production et de développer son expertise ouvrent de nouvelles opportunités pour les acteurs du secteur agro-alimentaire (Gow et al, 2002; 2003). Ceci demande aux acteurs de mettre en avant et d'exploiter leurs compétences clefs et de coopérer avec d'autres entités qui possèdent des connaissances différentes nécessaires au développement des activités, par exemple dans le domaine de la commercialisation ou de la transformation. Ce processus complexe montre que les producteurs peuvent être au cœur du processus de création de valeur s'ils savent jouer un rôle prépondérant au sein du secteur. Selon Goldsmith et Gow (2005), la structure de gouvernance mise en place ne crée pas de valeur en soit mais elle est propice à la création de valeur. Ainsi, si le choix de la coordination verticale au sein de la chaîne semble permettre à une organisation de producteurs de capter l'ensemble des bénéfices, il n'en est pas moins créateur de fortes contraintes managériales. S'orienter vers la quasi-intégration passant par des alliances avec des partenaires stratégiques voire par la création de «netchains ${ }^{6}$ » permet de limiter ces contraintes et d'avoir un accès rapide et direct à l'information.

\footnotetext{
4 Les NGC se distinguent des coopératives «traditionnelles » en ayant un nombre restreint de membres qui possèdent des droits de livraison spécifiques basés sur le nombre d'actions qu'ils possèdent. Ces actions, qui peuvent être cédées, déterminent le revenu des participants de la NGC et leurs donnent droit à un mode d'expression démocratique.

$5 \quad$ Le terme « hybrides» est repris de Ménard (2007).

$6 \quad$ Les «netchains » sont des réseaux complexes qui relient horizontalement et verticalement les producteurs aux fournisseurs d'intrants, aux industriels ainsi qu'aux consommateurs (Lazzarini et al, 2001).
} 


\section{II.4. Quel rôle pour l'état?}

31. Les considérations liées aux préoccupations des consommateurs et aux opportunités de création de valeur associées au sein du secteur agro-alimentaire concernent principalement des acteurs privés : les producteurs, les industriels et la grande distribution. Cependant, au sein des pays de l'OCDE, le secteur agricole est caractérisé par une forte implication de l'état. L'état peut jouer un rôle dans la détermination de l'ensemble de possibilités offert par l'évolution des attentes des consommateurs. Il peut soutenir le développement de certains labels en prônant la mise en place de cadres adaptés publics ou privés. Ces labels doivent être compréhensibles et fiables. Crespi et Marette (2003) décrivent les problèmes potentiels associés aux labels publics en particulier en matière de coûts. L'état peut aussi promouvoir le développement de modes de commercialisation alternatifs en facilitant leurs mises en place et les contrôles nécessaires. Par ailleurs, le développement de structures de gouvernance flexibles et évolutives n'est possible que si le cadre légal est adapté.

32. L'état peut aussi encourager la coopération entre les acteurs du secteur. C'est ce qui se passe au Royaume-Uni. Depuis la crise de la fièvre aphteuse, il existe là-bas un certain intérêt pour comprendre comment les producteurs pourraient créer et recevoir plus de valeur au sein du système agro-alimentaire. La «Sustainable Farming and Food Strategy ${ }^{7}$ » a été conçue sur la base du Rapport Curry ${ }^{8}$ qui appelait à reconnecter la chaîne agro-alimentaire avec ses clients. Elle vise à rapprocher les producteurs, les industriels et la grande distribution et a pour organe d'implémentation l'organisation indépendante English Farming and Food Partnerships ${ }^{9}$ (EFFP) qui travaille directement avec des organisations de producteurs et des firmes agro-alimentaires volontaires.

33. EFFP donne des conseils pratiques pour aider les acteurs à créer de la valeur en cherchant par exemple à développer des solutions industrielles répondant aux besoins des consommateurs ; solutions qui sont basées sur la coopération entre les acteurs de la chaîne. EFFP s'intéresse en particulier à l'approvisionnement du secteur public et à la promotion de la production et de la commercialisation de produits locaux. EFFP travaille ainsi avec la coopérative de producteurs laitiers Dairy Farmers of Britain ${ }^{10}$ (DFOB) sur le renforcement de l'organisation de la coopérative en mettant en place des formations et des stages d'étude. Ceci doit permettre à chaque membre de DFOB d'améliorer la profitabilité de sa production et d'accompagner DFOB dans ses choix stratégiques, à savoir les investissements dans des structures de transformation et dans le développement de produits laitiers et des marques associées.

\section{II.5. Quelles perspectives pour les producteurs?}

34. Le succès naissant de la marque de lait frais «Localchoice $»^{11}$, une marque lancée par Tesco en collaboration avec DFOB en Mai 2007 laisse envisager le potentiel d'une collaboration accrue au sein de la chaîne entre les producteurs membres de DFOB et la grande distribution. Les petites exploitations familiales de DFOB participantes à l'opération «Localchoice» se voient acheter leur production de lait

\footnotetext{
$7 \quad$ http://www.defra.gov.uk/farm/policy/sustain/index.htm.

$8 \quad$ Le rapport de la "Policy Commission on the Future of Farming and Food" (2002), présidée par Sir Don Curry, est communément nommé Rapport Curry. Il est disponible sous http://archive.cabinetoffice.gov.uk/farming/ Www.effp.com

10 DFOB compte 2600 exploitations membres en Angleterre et au Pays de Galles et emploie 2750 personnes. La coopérative possède 60 dépôts de distribution et 10 sites de transformation au Royaume-Uni.

Actuellement, 500000 litres de lait « Localchoice » sont vendus par semaine.
} 
avec un premium par Tesco ${ }^{12}$. Ce lait est conditionné par la coopérative à proximité des exploitations et ensuite vendu à un prix plus élevé que le lait «standard» de la marque de distributeur dans les magasins les plus proches sous la marque « Localchoice».

35. Cette initiative est intéressante à plus d'un titre. D'abord, elle souligne le potentiel de croissance des ventes de produits alimentaires produits localement et commercialisés comme tels. Ensuite elle montre comment l'ensemble de la chaîne agro-alimentaire peut en tirer partie. La marque "Localchoice » donne l'opportunité aux exploitations membres de DFOB participant à l'opération d'améliorer leurs revenus sans modifier les caractéristiques de leur production (donc à moindre coût) et à Tesco de mettre en avant son soutien au secteur laitier du Royaume-Uni et sa volonté de proposer à ses clients des produits « locaux ». Les structures d'organisation de DFOB et de Tesco permettent d'assurer que le lait vendu sous la marque « Localchoice » soit conditionné et commercialisé à proximité de l'exploitation.

36. L'exemple de la marque «Localchoice» montre que les producteurs peuvent utiliser des stratégies de différenciation pour créer et capter de la valeur même s'ils ne bénéficient pas d'économies d'échelle. En général pour que la stratégie puisse voir le jour, il faut que les producteurs soient organisés de manière collective et structurée. Les stratégies de différenciation des produits alimentaires « traditionnels » remportent un certain succès auprès des consommateurs et peuvent donc être utilisées sous une forme ou une autre pour permettre aux producteurs d'améliorer leurs revenus.

37. Quelles sont les perspectives de développement de ces stratégies à l'avenir? Pour un produit donné, les capacités d'expansion de la demande peuvent être limitées du fait de la faible élasticité prix de la demande pour la qualité ou pour d'autres aspects comme le respect de l'environnement. Mais l'information des consommateurs ainsi que l'anticipation de leurs besoins pourraient dans le futur amener de nouveaux segments de clients vers des produits «traditionnels » différenciés, produits qui sont souvent implicitement considérés comme élitistes.

38. Parmi les menaces à la viabilité économique des stratégies misant sur des produits «adaptés » à la demande des consommateurs, on trouve la nécessité de maintenir la confiance des consommateurs dans les systèmes de signalisation des caractéristiques des produits. Le rôle des organismes indépendants certificateurs qui vérifient que le cahier des charges est respecté est donc particulièrement important. Ceci souligne encore une fois le besoin de formes d'organisation adaptées.

39. Par ailleurs, les produits proposés pourraient devenir facilement imitables au niveau international du fait de l'augmentation de la qualité moyenne des produits. Les nouveaux acteurs dans les pays en développement utilisent des stratégies similaires avec par exemple la production biologique et le commerce équitable. S'il existe trop de produits différentiés, la portée des signaux envoyés aux consommateurs risque d'être limitée.

40. La mise en avant de caractéristiques spécifiques des produits «traditionnels » n'est pas la seule possibilité pour répondre à l'évolution des attentes de la société. Le recours à l'innovation avec le développement de nouveaux produits ou de nouvelles méthodes de production est une autre possibilité promise à un avenir certain. Les innovations et l'ensemble d'opportunités qu'elles contribuent à ouvrir sont le sujet de la deuxième section du rapport.

12 D’après DFOB (http://www.dfobmember.co.uk/DocFrame/DocView.asp?id=361\&sec=-1), le premium payé aux exploitations vendant leur lait sous un contrat «Localchoice » atteignait en moyenne 1 Pence par litre en Novembre 2007. 


\section{Innovations et création de valeur}

41. Avec la mondialisation des marchés, les entreprises du secteur agro-alimentaire vont être forcées d'opérer dans un contexte de plus en plus compétitif que ce soit en termes de prix ou en termes de développement de nouveaux attributs, nouveaux débouchés ou encore de nouveaux procédés. Ces nouveaux éléments devraient être le résultat d'innovations généralement élaborées loin des exploitations agricoles. Dans ce contexte, les entreprises du secteur ont déjà commencé à s'adapter et certaines remportent déjà un certain succès. Elles s'adaptent en étendant leur secteur d'activité par exemple par le biais d'alliances au sein du secteur ou avec des entreprises d'autres secteurs. Elles ont aussi mis en place de nouveaux modèles d'opération et de nouvelles formes d'organisation et de coopération.

42. Par ailleurs, les entreprises investissent dans de nouvelles technologies en matière d'équipement, de compétences et de procédés et modifient l'ensemble des techniques et des procédés utilisés pour améliorer la productivité et la profitabilité étant données les nouvelles configurations des marchés. Tous ces changements sont rendus possibles du fait d'activités innovantes ou de recherche et de développement (R\&D) ayant cours au sein des secteurs en amont et en aval des exploitations agricoles et bien souvent au sein des secteurs de l'économie n'ayant pas trait avec le monde agricole. Les innovations qui sont à la portée des producteurs peuvent leur permettre de rester compétitifs même si la demande et les marchés évoluent. Ainsi, les connections entre un secteur agricole compétitif et l'innovation pourraient être cruciales pour permettre à ce secteur de créer de la valeur ou plus simplement de rester compétitif.

43. Cette section du document discute des liens entre innovations et possibilités de création de valeur au sein du secteur agro-alimentaire et au niveau des exploitations agricoles en particulier. Elle est illustrée de quelques études de cas présentées en annexe. Dans un premier temps, elle présente une discussion du concept d'innovation au sein de l'économie et un cadre pour comprendre ce qui permet à des activités innovantes de voir le jour et les possibilités pour les gouvernements de soutenir ces activités. Dans un second temps, les nouvelles méthodes de production et les nouveaux produits en développement au sein du secteur seront présentés d'une manière non exhaustive. Ces nouveaux produits peuvent avoir pour objectif principal d'améliorer la santé ou de remplacer dans une certaine mesure certains produits issus de ressources non renouvelables.

\section{III.1. Comment se développent les innovations?}

44. L'innovation est couramment reconnue comme un des éléments essentiels à la croissance économique sur le long terme car elle participe à la création de nouveaux produits, de nouveaux procédés et de nouveaux marchés. Elle fait partie des activités du secteur privé qui sont régies principalement par la compétition au sein du marché. A l'inverse de la majorité des activités économiques, les coûts et bénéfices d'un certain investissement ne sont pas connus à l'avance. Les activités liées à l'innovation sont incertaines en termes de résultats et complexes. Elles dépendent de la recherche de base scientifique, de la connaissance accumulée et de l'expérience («Learning by doing ») (Dosi, 1988 ; Baptista et Swann, 1998). Cependant il est important de comprendre comment des innovations peuvent voir le jour lorsque des politiques sont mises en place pour chercher à promouvoir les activités innovantes menées par les entreprises.

45. L'économie néoclassique a souvent postulé une approche où l'augmentation de la demande joue un rôle fondamental («demand pull »). Les entreprises répondent à la demande nouvelle ou aux attentes des consommateurs pour des biens et des services n'existant pas encore. Les opportunités latentes en matière de bénéfices futurs guident les entreprises vers certains choix concernant le développement d'innovation ou les décisions d'investissement dans la $R \& D$. Ceci suppose qu'il existe un mécanisme pour reconnaître et comprendre a priori les besoins des consommateurs et un ensemble de technologies et de produits disponibles prêts à répondre à leur demande. L'approche selon laquelle l'offre ou la disponibilité de 
connaissances nécessaires («supply push ») sont le moteur des progrès technologiques ou des innovations est elle aussi simplificatrice : les dépenses en matière de recherche et développement donnent naissance à de nouveaux produits et procédés dont les consommateurs ont besoin et pour lesquels ils sont prêts à payer. Ces visions linéaires de l'innovation sont critiquées (Freeman et Louca, 2001 ; Teece, 1989).

46. Les déterminants spécifiques d'une innovation donnée sont souvent difficiles à identifier. Le développement d'innovations a souvent lieu lorsqu'une entreprise qui conduit son travail dans le cadre ordinaire s'aperçoit d'une anomalie. En effet, les entreprises mettent généralement en œuvre des routines prédéfinies lorsqu'elles initient des activités de R\&D. Ce sont les problèmes rencontrés et les travaux visant la résolution de ces problèmes qui indiquent les domaines où les ressources doivent être renforcées et les directions à prendre ou à éviter (Teece, 2008). Ces activités de routine sont des paradigmes technologiques qui guident les trajectoires choisies par la recherche (Dosi, 1982). Cependant, les entreprises cherchent aussi en dehors de ces paradigmes et, selon Teece, « des entreprises excellentes dans ce domaine cherchent aussi à identifier des opportunités et mettent en œuvre des technologies et des actifs spécialisés associés de manière à créer de «nouvelles combinaisons » ou des innovations ».

47. Même si les innovations sont développées au niveau de l'entreprise, elles opèrent dans un environnement économique et institutionnel qui est plus ou moins favorable aux activités de recherche. Ce cadre se situe au niveau national mais aussi aux niveaux régional et local comme présenté ci-dessous. Les activités de $\mathrm{R} \& \mathrm{D}$ des entreprises ont besoin de s'opérer dans un environnement propice pour mettre au point des innovations à la fois nécessaires et financièrement viables. Ces aspects ont été étudiés dans le cadre de systèmes nationaux d'innovation et dans le contexte de l'étude des performances comparatives des pays en matière de productivité de la R\&D (Nelson, 1993; Rosenberg, 1995; Stern et al., 2000).

48. Les recherches ont montré que même si les activités de R\&D sont dispersées tout autour de la planète, leur localisation semble être un des déterminants de la capacité des entreprises à créer de «nouvelles» avancées technologiques. Cette différence a été attribuée aux différences de capacités à innover des pays (Stern et al., 2000). Cette capacité à innover se définit comme l'aptitude d'un pays à produire et commercialiser un flux de technologies innovantes sur le long terme (Stern et al., 2000). Elle demande un ensemble interconnecté d'investissements et d'engagements politiques et de ressources qui soutiennent les nouvelles technologies et le caractère innovant de toute l'économie en général. Dans ce cadre, les entreprises peuvent bénéficier d'infrastructures économiques et institutionnelles favorables (Rosenberg, 1972 ; Nelson, 1993 ; Nelson et Rosenberg, 1994 ; Stern et al., 2000) ${ }^{13}$.

49. Des cadres similaires mais aux niveaux régional et local ont été suggérés comme étant nécessaires à la réflexion portant sur l'innovation. Dans cet environnement, des facteurs institutionnels plus locaux ainsi que des infrastructures locales et régionales peuvent structurer la capacité à innover dans un lieu donné (Rodriguez-Pose et Crescenzi, 2005). Cette capacité à innover au niveau local peut être mise en évidence par la présence de réseaux d'entreprises sur des territoires donnés souvent centrés sur un pôle scientifique et qui sont les initiateurs d'activités innovantes. Krugman (1991), d'une façon similaire à Becattini (1987), développe une théorie de spécialisation régionale des activités industrielles basée sur des pools de main d'œuvre de qualité et sur les biens intermédiaires ${ }^{14}$.

50. La caractéristique du réseau géographique (ou cluster) est sa capacité à générer des externalités positives. Ceci est du à différents facteurs: 1 - La création d'un pool de travailleurs qualifiés qui ont des compétences communes, 2- La disponibilité d'un ensemble d'intrants nécessaires et ce à un coût réduit

13 Les trois structures théoriques qui sous-tendent le cadre de la capacité nationale à innover sont : 1 - les systèmes d'innovation nationaux (Nelson, 1993), 2 - la croissance endogène portée par la connaissance (Romer, 1990) et 3- le paradigme de l'avantage concurrentiel dans l'industrie nationale (Porter, 1990). 
lorsque la demande s'accroit et 3- Des effets positifs (spillover effects) dus à l'échange d'information et d'expérience qui a lieu entre les entreprises du réseau. Ces effets induits qui prennent des formes formelles et informelles sont importants pour les activités innovantes car ils fournissent une infrastructure technologique (Baptista et Swann, 1998 ). Ceci signifie que les réseaux d'entreprises pour un secteur, une industrie ou des ressources donnés vont pouvoir utiliser ces externalités et fournir un environnement économique plus efficace et productif pour leurs opérations et les activités de R\&D.

51. Ces clusters innovants bénéficient clairement de la présence d'une forte capacité à innover au niveau national, niveau dans lequel ils opèrent. La capacité nationale à innover se reflète non seulement dans les niveaux de financement des activités de R\&D mais aussi dans le développement d'un capital humain grâce à des investissements plus importants dans le secteur éducatif. D'autres incitations au niveau local peuvent créer des synergies avec les politiques de type macroéconomique définies au niveau national pour améliorer les bénéfices apportés par les réseaux d'entreprises et les innovations. Le rôle du gouvernement est de continuellement renouveler le cadre favorable à la promotion des activités innovantes et d'améliorer la compétitivité des entreprises dans les clusters.

\section{III.2. Qu'en est-il pour le secteur agro-alimentaire?}

52. En général, les producteurs du secteur agricole ne s'engagent pas directement dans la recherche et le développement. Ils adoptent les innovations qui ont été générées dans d'autres secteurs de l'économie comme par exemple les secteurs de l'industrie chimique, des transports, ou des nouvelles technologies de l'information et de la communication. Ils bénéficient des augmentations de la productivité que ces innovations permettent ainsi que des possibilités d'accès à de nouveaux marchés ou de développement de nouveaux attributs pour les produits.

53. Tout comme il n'existe pas un processus de création de valeur au sein du secteur agroalimentaire, il n'existe pas une seule et unique voie pour l'innovation. Les innovations qui découlent de l'évolution des préoccupations de la société peuvent prendre de multiples formes: innovations sur les méthodes de production permises entre autres par la baisse des coûts des nouvelles technologies, innovations sur les produits fabriqués par le secteur avec en particulier le développement de nouveaux produits orientés vers la santé ou les utilisations non alimentaires. Les trois prochaines sous-parties donnent un aperçu non exhaustif des développements en cours au sein du secteur agro-alimentaire dans ces domaines et étudient les perspectives associées pour l'ensemble des acteurs et pour les producteurs en particulier.

\section{III.3. Nouvelles méthodes de production et création de valeur}

54. Développer de nouvelles techniques de production agricole répond à la fois aux attentes de la société en matière de durabilité environnementale et participe au processus de création de valeur pour les producteurs en aidant à une diminution des coûts de production. Le formidable essor des technologies de l'information et de la communication et le développement d'innovations associées s'appliquant au secteur de l'agriculture permettent d'envisager de profondes modifications des systèmes de production utilisés en agriculture.

55. Ainsi, l'agriculture de précision qui consiste en la gestion précise des parcelles agricoles en prenant en compte leurs spécificités agronomiques et climatiques se développe depuis plusieurs années. Elle utilise les nouvelles technologies comme les ressources informatiques et les informations fournies par des satellites pour optimiser la performance des cultures dans une démarche de préservation de l'environnement et d'augmentation des marges de production. En effet, elle permet par exemple de doser parfaitement l'utilisation de certains engrais ou de limiter l'irrigation ou bien encore de suivre l'évolution des rendements des plantes. 
56. Fountas et al. (2005) notent que le développement de l'agriculture de précision est freiné par le besoin d'apprentissage pour les producteurs concernés et les investissements nécessaires pour maitriser les techniques. Les nouvelles technologies dont les coûts continuent de diminuer offrent pourtant une palette importante de choix pour gagner en efficacité opérationnelle et pour améliorer l'impact environnemental de la production agricole.

57. L'étude de cas A-II. présentée en annexe du rapport donne un exemple de l'utilisation de techniques d'irrigation au goutte à goutte et de fertirrigation associées à des technologies nucléaires pour permettre d'augmenter la valeur créée lors de la production de pommes de terre en Turquie et aussi de minimiser l'impact environnemental de cette production. Cette étude de cas montre que l'utilisation à bon escient de nouvelles techniques en apparence très éloignées du secteur agricole peut avoir un double objectif, un objectif économique et un objectif environnemental, mais demande une forte implication des acteurs concernés.

58. Le développement de nouvelles techniques de production nécessite souvent d'importants investissements financiers en matière de recherche et développement. Couramment, comme c'est le cas pour l'exemple turc présenté dans l'étude de cas A-II., le financement de tels développements ou de leur adoption par les producteurs a besoin d'être pris en charge au moins partiellement par des fonds publics ou par des partenariats publics-privés.

59. Le rapport de l'OCDE (2008b) souligne qu'il reste beaucoup à faire pour améliorer substantiellement l'efficacité de la production agricole d'un point de vue environnemental. Moins de la moitié des pays de l'OCDE a mis en place un suivi des changements en termes de pratiques de gestion environnementale des exploitations agricoles. Dans ce contexte, la coopération des états au développement de nouvelles méthodes de production agricole et à leur adoption par les producteurs - qui pourrait par exemple prendre la forme de fonds pour la recherche, de crédits et de formations pour les producteurs adoptant ces nouvelles techniques - pourrait accélérer l'évolution du secteur vers une meilleure prise en compte des problèmes environnementaux et pourrait permettre aux producteurs de modifier leurs coûts de production.

60. Dans la majorité des cas, les producteurs ne seront pas les développeurs des innovations permettant à de nouvelles méthodes de production de voir le jour. Ils devront donc adopter ces innovations et ce avec un certain coût financier et d'apprentissage. C'est le rapport perçu entre coûts et bénéfices qui déterminera les choix des producteurs.

\section{III.4. Bioéconomie et création de valeur}

61. Les producteurs peuvent-ils participer directement au nouveau paradigme de la création de valeur? En principe, il semble exister une place certaine pour des clusters innovants se concentrant sur l'agriculture au niveau régional. Ces clusters peuvent être orientés vers les produits agro-alimentaires traditionnels mais les opportunités de création de valeur semblent plus importantes si les produits agricoles sont valorisés entièrement.

\section{III.4.1. Innovations et bioproduits}

62. En effet, la demande croissante pour les produits issus de ressources renouvelables en particulier à des fins non alimentaires est un moteur puissant d'innovation et de progrès technique au sein du secteur agro-alimentaire. Depuis quelques années, de nombreux bioproduits sont en cours de développement. La société les considère comme des moyens potentiels d'encourager la durabilité environnementale et industrielle. La notion de bioéconomie pour qualifier le développement des bioproduits est apparue au 
cours des dernières années et fait l'objet d'un projet majeur du Programme de l'OCDE pour l'Avenir (IFP) : « La Bioéconomie à l’Horizon $2030 »$.

63. Le rapport de l'OCDE (2006) définit la bioéconomie comme l'ensemble agrégé des opérations économiques qui utilisent la valeur latente en provenance de produits et procédés d'origines biologiques pour capter de nouveaux bénéfices en termes de croissance et de bien-être pour les citoyens et la société en général. Ces bénéfices dérivent des gains de productivité induits, des effets sur la santé de certains des bioproduits, des effets de substitution par rapport aux technologies existantes ou encore de l'utilisation durable et respectueuse des ressources.

64. La bioéconomie est rendue possible par l'avancée rapide des connaissances scientifiques et techniques. Ce dynamisme est réalisable du fait de la baisse des coûts liés à la recherche et au développement, de la variété des applications de la bioéconomie, de la grande implication de l'ensemble des acteurs de la société du simple citoyen au gouvernement en passant par les grands groupes industriels et les producteurs du secteur agricole. Dans ce contexte, les nouvelles technologies augmentent l'ensemble des possibilités du secteur agricole.

65. Le développement de la production de biocarburants de $1^{\text {ère }}$ génération au cours des cinq dernières années est l'illustration de l'essor des bioproduits. Les politiques incitatives des pays membres de l'OCDE ont contribué à ce développement et au niveau élevé des prix des matières premières probablement appelé à se maintenir au cours des prochaines années (OCDE, 2008a; OCDE, 2008c). Le développement de la $1^{\text {ère }}$ génération de biocarburants tend à poser les utilisations non-alimentaires de produits agricoles en rival des utilisations alimentaires. Ceci pourrait être différent dans le futur avec le développement de nouveaux produits à destination non-alimentaire dans le cadre de la bioéconomie.

\section{III.4.2. Nouvelles opportunités de création de valeur}

66. Avec les améliorations des procédés de bioraffinerie, c'est-à-dire des procédés de transformation de l'ensemble de la biomasse, les produits végétaux pourraient être utilisés dans leur globalité : utilisations alimentaire et non alimentaire pourraient être couplées. Les productions de base du secteur agricole pourraient être transformées en un ensemble de produits comme de la nourriture, de l'essence, des matériaux de constructions ou des produits utilisés pour l'industrie chimique « verte ». 
TAD/CA/APM/WP(2008)15/FINAL

\begin{abstract}
Encadré 1. Quelques perspectives associées au développement des biocarburants de $2^{\text {ème }}$ génération
Le rapport de l'OCDE (2008a) décrit en détail les perspectives associées au développement des biocarburants de $2^{\text {ème }}$ génération.

\section{Quelle technologie?}

La technologie de production des biocarburants de $2^{\text {ème }}$ génération est en cours de développement. Elle repose sur la raffinerie de la biomasse. Des installations pilotes et de démonstration sont en création ou en phase de test en Allemagne, au Canada, aux États-Unis ou encore en France. La technologie finale qui sera adoptée à grande échelle dans le futur dépendra des résultats de ces tests. Actuellement deux technologies sont en concurrence: l'éthanol cellulosique et le Biomass to Liquid (BtL). Les opportunités de création de valeur associées pour le secteur agricole ne sont pas encore connues mais il est probable qu'elles soient non négligeables.

Étant données les préoccupations de la société pour les questions liées au changement climatique et à l'environnement, la définition, le développement et la mise en fonction de la technologie des biocarburants de seconde génération ne peut pas prendre en compte uniquement les questions de rentabilité économique et de création de valeur pour les différents acteurs impliqués mais aussi leurs performances écologiques.

\section{Où produire les biocarburants de $2^{\text {nde }}$ génération ?}

Si la biomasse utilisée est d'origine agricole ou forestière, l'implantation d'installations de production ne sera sans doute possible qu'à proximité de régions à vocation fortement agricole ou forestière. En effet, les intrants de ces usines seront en provenance directe d'exploitations agricoles ou de forets ou encore d'usines de transformation de matières premières agricoles. Les producteurs seront donc des fournisseurs privilégiés et devraient bénéficier de ce statut. Bien entendu, la rentabilité des bioraffineries une fois la technologie au point dépendra de leurs zones d'implantation et des économies d'échelle.

\section{Quelles perspectives pour les producteurs?}

Si les nouvelles technologies associées aux biocarburants de $2^{\text {nde }}$ génération permettent l'utilisation de déchets agricoles qui n'avaient pas ou peu d'utilisation, un nouveau marché pourrait être développé. Les producteurs agricoles pourraient revendre des matériaux qui jusqu'ici étaient au mieux sans valeur et pour lesquels il fallait parfois payer pour se débarrasser. Pour les producteurs, la question des opportunités d'une utilisation alternative de ces déchets se pose. En effet, les déchets agricoles peuvent être utilisés dans une certaine mesure comme engrais organique.

Par ailleurs, si des terres à moindre rendement sont utilisées pour produire des variétés d'arbres présentant de courts cycles de développement ou des produits agricoles peu communs comme le switchgrass ou le miscanthus, les opportunités de création de valeur pourraient être importantes pour les producteurs. Ces terres seraient alors utilisées uniquement à des fins non-alimentaires, avec des effets incertains sur les prix des matières premières alimentaires. Un des problèmes potentiels au développement de ces nouvelles cultures est le coût d'installation : les premières années, ces terres ne rapporteraient rien ou presque. De plus, un système de rotation des cultures adéquat devrait être défini pour limiter entre autres les impacts sur la biodiversité.
\end{abstract}

67. La deuxième génération de biocarburants devrait être basée sur les technologies de transformation de la biomasse. La biomasse utilisée pourrait prendre la forme de déchets agricoles ou forestiers, de variétés spécifiques d'arbres ou de plantes qui seraient cultivées sur des terres agricoles à moindre rendements ${ }^{15}$, mais aussi de déchets organiques ou d'algues. L'Encadré 1 décrit brièvement certaines perspectives associées au développement des biocarburants de $2^{\text {ème }}$ génération. Bien-entendu, les utilisations non-alimentaires de produits agricoles ne se résument pas aux biocarburants de première et deuxième générations. Les bioproduits comme les matériaux de construction naturels, les solvants

15 Les terres agricoles à moindre rendements peuvent demander l'utilisation de méthodes de productions moins intensives pour empêcher la dégradation des sols et les perturbations d'écosystèmes potentiellement fragiles. 
d'origine végétale et toute la chimie verte se développent à vitesse rapide. Ce développement demande aux acteurs du secteur agro-alimentaire d'innover profondément avec le développement de nouvelles technologies.

68. Le développement de procédés innovants est illustré dans l'étude de cas A-III. qui traite du pôle de compétitivité français Industries et Agro-Ressources. La société ARD, qui fait partie de ce pôle, développe entre autres des brevets liés à la chimie du végétal souvent en partenariat avec des acteurs de la recherche publique et des industriels. Sa filiale Soliance est chargée de la production et de la commercialisation des produits associés.

\section{III.4.3.Vers des réseaux permettant aux acteurs du secteur de tirer partie de l'innovation}

69. Les producteurs agricoles peuvent tirer partie du développement de ces produits à forte valeur ajoutée s'ils sont parties prenantes du processus d'innovation technologique. Ceci n'est possible que grâce à des formes d'organisation adaptées. Dans le cas d'ARD, les producteurs par l'intermédiaire de leurs coopératives sont actionnaires de l'entreprise de R\&D.

70. Par ailleurs, si les utilisations non-alimentaires des produits agricoles passent par une valorisation de l'ensemble du produit, il semble nécessaire que l'ensemble des activités de transformation du même produit initial aient lieu dans des industries localisées dans des zones proches, ce pour diminuer voire annuler les coûts de transport et de stockage entre les différentes étapes de transformation. Cette nécessité est aussi illustrée dans l'étude de cas A-III. avec le site de Pomacle dans la Région Champagne Ardenne en France où l'ensemble des activités liées à la transformation de betteraves à sucre et de blé à des fins alimentaires et non-alimentaires est concentré sur une même zone.

71. Le pôle de compétitivité français Industries et Agro Ressources allie innovation sur les produits et innovation en matière de formes d'organisation. Il existe en effet un réseau d'acteurs interconnectés regroupant recherche publique, entreprises de $R \& D$, industrie et coopératives d'agriculteurs. Il illustre le fait que le développement de nouveaux produits est souvent accompagné de structures d'organisation innovantes. La recherche portant sur les stratégies de compétition a montré que les entreprises qui ont du succès tendent à s'agglomérer géographiquement à proximité des centres de recherche. Sur une certaine période, ces effets peuvent être cumulatifs à cause des retombées et des synergies qui sont générées. Il est très difficile de prévoir le futur des clusters qui misent sur la bioéconomie car ces réseaux sont en cours de formation.

72. Une chose est certaine : ces réseaux devraient être amenés à se développer dans des régions à vocation agricole et pourraient apporter des retombées économiques pour les agriculteurs et l'économie locale en termes de revenu et d'emploi du fait du développement de produits associés ou des besoins induits par les industries de transformation. C'est dans ce contexte que le rôle potentiel de l'état pour soutenir la mise en place de clusters et d'activités innovantes se comprend le mieux. Dans le cas du pôle de compétitivité français Industries et Agro-Ressources, l'état est un acteur important car il finance une partie des projets d'innovations. Grâce aux nouvelles technologies de l'information et de la communication, la localisation des activités liées au développement d'innovations pourrait être amenée à jouer un rôle moins important. Un «réseau» peut se développer à distance. Ainsi, par exemple, le pôle de compétitivité Industries et Agro-Ressources collaborent avec des centres de recherche et des entreprises situés dans d'autres régions françaises ou à l'étranger,

\section{III.5. Innovations et aliments fonctionnels}

73. L'évolution des attentes de la société demande aussi au secteur agro-alimentaire de développer de nouveaux produits qui peuvent avoir pour fonction d'améliorer la santé des personnes qui les consomment. 
Le terme « aliments fonctionnels ${ }^{16}$ » désigne les aliments et les composants alimentaires susceptibles d'être plus bénéfiques à la santé que les aliments ordinaires. Cela fait déjà longtemps que l'industrie alimentaire a perçu l'opportunité de fournir aux consommateurs des produits alimentaires présentant certaines caractéristiques nutritionnelles spécifiques en particulier en matière d'impact sur la santé.

\section{III.5.1. Développement du marché des aliments fonctionnels}

74. Née il y a vingt ans avec une margarine anti-cholestérol lancée par Unilever, la filière des aliments fonctionnels brasse aujourd'hui 75.5 milliards USD dans le monde (Weststrate et al., 2002). Elle a étendu sa gamme bien au-delà des margarines et est désormais présente dans tous les domaines du secteur agroalimentaire. Les produits enrichis en oméga- ${ }^{17}$ font partie des aliments fonctionnels les plus représentés. L'étude de cas A-IV présente les finalités et le développement de ces produits. A l'heure actuelle, beaucoup d'efforts sont déployés pour mettre sur le marché toute une gamme d'aliments fonctionnels, d'alicaments voire même de nouveaux médicaments synthétisés à partir de plantes.

75. Comme le souligne le rapport d'Agriculture et Agro-Alimentaire Canada (2002) sur les bénéfices potentiels des aliments fonctionnels, le marché des aliments fonctionnels va être amené à se développer avec la montée des préoccupations liées à la santé au sein des pays de l'OCDE. La demande d'alicaments ou d'aliments fonctionnels est à la fois un défi et une opportunité pour le secteur alimentaire. Si les innovations peuvent être adaptées de manière à répondre aux nouvelles demandes et à les anticiper, elles peuvent profiter aussi bien aux exploitants, à l'industrie et aux consommateurs.

76. Dans ce contexte, le Nordic Innovation Center ${ }^{18}$, instrument du Conseil Nordique des Ministres pour promouvoir l'innovation et l'intelligence économique dans le secteur industriel des pays nordiques, a lancé en 2006 une initiative concernant le secteur des aliments fonctionnels. Selon ce centre sur l'innovation, les aliments valorisés pour leur impact sur la santé offrent une palette d'opportunités pour les producteurs agricoles locaux. Ainsi, une série de projets destinés à aider l'industrie agro-alimentaire nordique à s'imposer comme un participant compétitif dans le domaine des aliments fonctionnels a été lancée en 2006.

77. Le projet Grainity ${ }^{19}$ concerne un partenariat entre secteur public et secteur privé qui traite du potentiel santé des céréales complètes (blé complet, seigle et avoine) et de sa valorisation auprès des consommateurs locaux ou au sein de marchés d'exportation (États-Unis, Royaume Uni). Il capitalise sur le succès de l'initiative « 6 fruits et légumes par jour » au Danemark pour lancer une campagne comparable sur le bienfait des céréales complètes, céréales beaucoup plus consommées dans les pays du nord de l'Europe que dans les autres régions du monde. Cette campagne se base sur une coopération entre les partenaires commerciaux (producteurs de céréales, industriels et grandes surfaces) et les partenaires santé (gouvernements et chercheurs). Les partenaires santé donnent la légitimité et la caution scientifique ${ }^{20}$; les partenaires commerciaux s'organisent pour pouvoir en tirer partie.

16 La Fondation du Conseil International d'Information sur l'Alimentation (IFIC Foundation) donne des informations spécifiques sur les aliments fonctionnels : http://www.ific.org/nutrition/functional/index.cfm.

Les oméga-3 DHA sont un type d'acide gras polyinsaturé.

http://www.nordicinnovation.net .

Le site internet du projet Grainity est le suivant : http://www.vtt.fi/proj/grainity/.

Pour plus d'informations sur les effets des céréales complètes sur la santé, se référer au site internet: http://www.oatsandhealth.org/ . 
78. Préalablement à sa commercialisation en tant qu'aliment fonctionnel, chaque produit doit être reconnu conforme aux réglementations officielles. Dans la mesure où les réglementations en sont encore au stade de la conception, l'utilité publique des normes sanitaires est souvent synonyme d'obstacle au développement d'aliments fonctionnels innovants. Les homologations ou certifications officielles influencent souvent favorablement l'opinion publique. Cependant, comme tout nouveau produit, la plupart des aliments fonctionnels commercialisés ne réussit pas à percer sur les marchés. C'est le cas par exemple de la marque de produits VITO développée par les Petites Fermes du centaure au Canada et homologuée par l'état qui est présentée dans l'étude de cas A-IV.

\section{III.5.2. Acceptation par les consommateurs}

79. Le succès de la mise sur le marché des aliments fonctionnels dépend en grande partie de l'acceptation par les consommateurs du message liant l'aliment fonctionnel à son objectif santé. Ce processus d'acceptation diffère selon les pays (Labrecque et al, 2006) et dépend des preuves scientifiques (et de leur crédibilité) apportées par les industriels (Roberfroid, 2002 ; Frewer et al, 2003 ; Sibbel, 2006) ainsi que des systèmes légaux en place.

80. La question de l'acceptation se pose par exemple avec la mise sur le marché probable sous peu d'organismes génétiquement modifiés susceptibles d'avoir des effets sur la santé ou sur la durabilité environnementale comme illustré dans l'étude de cas A-V.. Cette étude de cas traite du travail d'un institut de recherche australien le CSIRO concernant des oléagineux enrichis en oméga-3 dont l'huile végétale obtenue serait utilisée comme nourriture pour les poissons d'élevage afin d'améliorer leurs caractéristiques nutritionnelles et de permettre la durabilité de la filière halieutique. Les recherches sur ces nouveaux oléagineux sont en cours et les investissements sont très importants bien que la phase de commercialisation dans le futur ne soit pas aisée.

81. Depuis l'introduction de la première génération d'OGM dont les principaux bénéfices visaient les producteurs agricoles (avec des rendements élevés ou des moindres coûts par exemple), ces plantes sont au cœur d'un débat qui concerne la santé et l'environnement. Dans la mesure où les nouveaux OGM qui seront développés dans un futur proche auront des objectifs principaux différents - probablement plus proches des attentes des consommateurs - il est légitime de s'interroger sur l'éventualité d'une meilleure acceptation de ces OGM par les consommateurs et par les états eux-mêmes au travers de leurs systèmes légaux (Giannakas and Yiannaka, 2008).

82. Les investissements dans la recherche offrent des marges de croissance importantes au marché des aliments fonctionnels dans le futur. A titre d'exemple, des chercheurs ont réussi à produire du lait et des produits laitiers enrichis en acide linolénique conjugué (Coakley et al., 2007).

\section{III.5.3. Quelles perspectives pour les acteurs du secteur et les producteurs en particulier?}

83. Le marché des aliments fonctionnels étant vraisemblablement amené à un développement rapide, les acteurs impliqués devront sans doute continuellement s'adapter aux nouveaux intrants et aux nouvelles méthodes de production et de transformation pour maintenir leur position. Le développement d'innovations en matière d'attributs santé des aliments concerne les producteurs agricoles en particulier.

84. Ceci est le cas lorsque les producteurs modifient leurs méthodes de production, comme illustré dans l'étude de cas A-IV. avec les exemples de l'Association Bleu-Blanc-Cœur et de la marque Vito. Dans ces deux cas, l'alimentation du bétail est modifiée pour que la viande commercialisée présente une plus forte teneur en oméga-3. Le succès de la commercialisation de ces produits avec un premium sur les produits standards dépend à la fois de la valorisation par les consommateurs des caractéristiques de ces nouveaux produits et de la structure d'organisation de la chaîne. Les producteurs souhaitant «seuls » 
mettre sur le marché de nouveaux produits risquent d'être confrontés à des difficultés, difficultés illustrées par le cas de la marque VITO. Les producteurs organisés avec d'autres acteurs de la chaîne, comme dans le cas de l'association Bleu-Blanc-cœur, semblent mieux tirer partie des opportunités créées par ces nouveaux produits.

85. Les efforts en matière d'innovation peuvent aussi porter sur la modification des caractéristiques de certains aliments lors de la transformation des produits. Dans l'étude de cas A-IV., le lait pour bébé Humana de la formule standard associe lait et huiles végétales par exemple. Dans ce contexte, la capacité pour les producteurs de tirer partie de la mise sur le marché d'aliments fonctionnels dépend de leur implication au sein de la chaîne de valeur. S'ils ne sont que de simples fournisseurs de produits agricoles standards, ils n'auront que peu de chance de créer de la valeur supplémentaire.

86. Par ailleurs, l'innovation peut porter sur le développement de nouveaux intrants (de nouvelles semences par exemple) qui permettront d'obtenir des matières premières agricoles présentant des caractéristiques particulières. C'est le cas des oléagineux enrichis en oméga-3 développés par le CSIRO présentés dans l'étude de cas A-V.. La manière dont le producteur lui-même pourra capter les bénéfices de ce type d'innovation dépendra des structures financières et légales gouvernant les relations commerciales entre les producteurs, les fournisseurs d'intrants et le reste de la chaîne. Du fait de l'ajout de caractéristiques spécifiques au produit agricole, la valeur de ce produit devrait augmenter et donc augmenter les revenus potentiels des producteurs.

87. Cependant, les coûts associés aux intrants, aux technologies de production et de transformation pourraient être importants. Ainsi par exemple, lorsque les intrants sont protégés par des brevets comme cela pourrait être le cas pour des semences aux caractéristiques spécifiques, le coût de ces intrants serait plus élevé. Pour le producteur agricole, il serait nécessaire que l'augmentation de la valeur du produit final couvre au moins cette augmentation des coûts des intrants.

88. Dans une certaine mesure, la gestion de certains types d'aliments fonctionnels pourrait présenter des caractéristiques similaires aux produits issus d'organismes génétiquement modifiés de $1{ }^{\text {ère }}$ génération avec des coûts plus élevés pour les intrants, une ségrégation au moment de la récolte et du stockage et dans les phases de transport et de transformation.

89. Pour résumer, le rapport d'Agriculture et Agro-Alimentaire Canada (2002) établit que les fournisseurs de matières premières peuvent espérer recevoir une part relativement plus faible de la valeur créée sur la chaîne dans le cas de l'industrie des aliments fonctionnels par rapport à l'industrie agroalimentaire conventionnelle (entre 5 et $25 \%$ contre de 10 à $70 \%$ pour les produits conventionnels). Ceci reflète le fait que la valeur au sein de la chaîne est souvent créée lors d'autres étapes, en particulier lors des phases de recherche et de développement de la technologie.

90. Ce sont les formes d'organisation de la chaîne de valeur et leur capacité à innover et à s'adapter aux changements qui déterminera si les producteurs pourront tirer pleinement profit du développement de ce secteur. Le développement des aliments fonctionnels comme de tout nouveau produit peut ainsi dans une certaine mesure être synonyme de création de valeur au sein du secteur agro-alimentaire.

\section{Vers un secteur dynamique participant à la croissance}

91. Le soutien agricole étant en diminution et les restrictions aux échanges tendant à disparaitre, le secteur agro-alimentaire se doit de s'adapter à un nouvel environnement économique. Il est donc poussé vers une redéfinition de ses activités à long terme. Les nouvelles attentes des consommateurs et de la société en général entrainent une modification des pratiques des producteurs passant par la mise au point 
de nouveaux produits répondant à la demande et par la modification des structures de commercialisation et des méthodes de production par exemple.

92. Pour être en mesure de rester compétitifs dans ce nouvel environnement et de rester en phase avec les attentes des consommateurs, les acteurs du secteur agro-alimentaire et plus particulièrement ceux du secteur agricole doivent continuellement mettre à jour l'ensemble de leurs produits et des techniques utilisées. Ils doivent aussi être capables de tirer à leur avantage les opportunités offertes par un monde où les développements technologiques sont rapides. Pour ce faire, le secteur doit innover. Le processus d'innovation au sein du secteur agro-alimentaire n'est pas un processus linéaire. Le secteur agroalimentaire peut tirer partie d'innovations développées dans d'autres secteurs de l'économie. Il peut aussi investir dans les activités de recherche et développement. Les opportunités de création de valeur associées sont importantes et elles peuvent aller au delà du secteur agro-alimentaire en créant par exemple des emplois dans des zones à forte vocation agricole.

93. Les stratégies en cours de développement dans le domaine de l'organisation et de la collaboration entre les différents acteurs du secteur agro-alimentaire ont un potentiel substantiel pour permettre au secteur d'innover ou de créer de la valeur. Dans ce contexte, le rôle des gouvernements peut être de mettre en place les paramètres en termes de structure légale et institutionnelle. Ils peuvent aussi être amenés à fournir des services au niveau régional ou local, des structures éducatives, des plateformes de recherche en partenariat entre le secteur public et privé, des formations et des services annexes. Ces infrastructures peuvent être le point de départ au développement de pôles de services, d'entreprises et de plateformes de recherche ou de formation qui sont l'essence des clusters. L'exemple du pôle de compétitivité Industries et Agro-Ressources montre que la mise en place de clusters associant producteurs, industriels et recherche publique ou privée peut être favorable à la création de valeur au sein du secteur et permet aux producteurs agricoles eux-mêmes de faire partie de ce processus.

94. L'innovation et la croissance sont caractérisées par un processus de destruction créative, où de nouvelles technologies, de nouveaux marchés et de nouvelles formes d'organisation renouvellent les anciens modes de fonctionnement des secteurs économiques. Il convient dans ce contexte de souligner le rôle possible du gouvernement pour que le processus d'adaptation soit moins délicat tout en faisant en sorte que l'innovation et la croissance continuent d'accroître le bien-être de tous. Le système légal luimême peut encourager l'innovation et le passage du secteur agricole vers un secteur plus dynamique d'un point de vue économique. Ainsi, il existe un rôle pour l'état d'aider à concevoir ou à mettre en place de nouveaux cadres réglementaires. Certains acteurs du secteur agro-alimentaire, et sans doute en particulier certains producteurs agricoles, risquent en effet de souffrir s'ils n'arrivent pas à s'adapter au nouvel environnement économique. Les initiatives politiques doivent donc dorénavant être conçues dans une optique d'accompagnement du développement du secteur dans son entier pour aider à la construction d'un environnement adapté à des acteurs flexibles et dynamiques.

95. Pour finir, il existe de nombreuses opportunités de création de valeur au sein du secteur agroalimentaire créées par les nouvelles attentes de la société et le nouvel environnement économique. Le rapport a proposé un panorama non exhaustif de ces possibilités avec en particulier le développement de nouveaux produits comme les bioproduits et les aliments fonctionnels. Cependant les risques sont élevés que la majorité de la valeur créée grâce à ces nouveaux débouchés ne soit pas ou peu captée par les producteurs eux-mêmes s'ils ne sont pas organisés collectivement et s'ils ne travaillent pas en partenariat avec le reste de la chaîne. Les producteurs qui ne bénéficient pas d'économies d'échelle et qui ne sont pas intégrés au sein de chaînes de valeur misant sur de nouveaux débouchés peuvent aussi tirer partie du nouvel environnement économique. Ceci passe par la différenciation de leur production, par le développement de nouveaux modes d'organisation au sein de la chaîne ou encore par la mise en place de nouveaux réseaux de commercialisation. 
96. En conclusion, le secteur agro-alimentaire est en train de vivre de profonds bouleversements. Ces changements permettent à tous les acteurs de redéfinir leurs méthodes de travail et les finalités de leur travail. Les possibilités sont importantes et devraient permettre au secteur de participer au dynamisme de l'économie. Pour les producteurs agricoles en particulier, il existe de nombreuses voies pour tirer partie de ces bouleversements. Pour créer et capter de la valeur, les producteurs doivent valoriser leur rôle au sein de la chaîne. Ceci passe par des structures d'organisation solides et par des choix clairs en matière d'orientation de la production en réponse à la demande des consommateurs. 


\section{ANNEXE : LES ÉTUDES DE CAS}

97. L'annexe présente cinq études de cas qui illustrent les propos développés dans le rapport en donnant des exemples d'initiatives permettant au secteur agro-alimentaire de créer de la valeur. Dès que possible, l'accent a été porté sur la façon dont les producteurs concourent au processus de création et de captation de valeur et sur la participation éventuelle de l'état. Ces exemples ont été choisis au sein de pays de l'OCDE. Les études de cas sont fournies à titre indicatif et illustratif. Elles ne se sont en aucun cas mises en avant comme des exemples à suivre ou non. La rédaction de cette annexe a été rendue possible grâce à la collaboration avec des personnes directement impliquées dans ces projets. Elles sont remerciées pour l'aide apportée.

\section{A-I. Le développement de circuits alternatifs de distribution alimentaire (Japon)}

98. Mr Kobayashi, chercheur principal au sein de l'Institut pour la Recherche Politique du Ministère de l'Agriculture, de la Forêt et des Pêcheries au Japon, et Mr Kimura, analyste au sein de la direction des Échanges et de l'Agriculture de l'OCDE, sont remerciés pour l'aide apportée à la préparation de cette étude de cas.

\section{Le mouvement des Teikei est apparu dans les années 1970}

99. L'apparition du mouvement alimentaire alternatif a d'abord été le fait pour l'essentiel des consommateurs, qui demandaient des produits locaux et des relations directes avec les producteurs. En 1971, un groupe d'agriculteurs, de consommateurs et de chercheurs a créé le mouvement Teikei (partenariat) pour encourager l'agriculture durable en s'appuyant sur des partenariats entre producteurs et consommateurs. Loin de se contenter d'acheter les produits, les consommateurs participaient parfois aux travaux agricoles. Le mouvement Teikei était limité à des groupes particuliers de personnes qui avaient en commun un même souci de santé et de protection de l'environnement. A ce titre, il ne constituait pas un circuit alternatif de commercialisation à la portée de la majorité des producteurs de denrées alimentaires.

\section{Les Sanchoku se développent depuis les années 1980}

100. Dans les années 80, les consommateurs n'aspiraient pas uniquement à trouver des produits bon marché et standardisés : ils cherchaient aussi des produits particuliers, par exemple issus de l'agriculture biologique. C'est ainsi que se sont développés les mouvements de Sanchoku (vente directe), à l'instigation de coopératives de consommateurs, de coopératives agricoles locales et de supermarchés. Tandis que le marché de gros ordinaire distribue des produits standardisés, les groupes de Sanchoku commercialisent en général des produits qui ont des caractéristiques particulières en termes de qualité et de variété. Dans ce système, producteurs et détaillants, restaurants ou transformateurs de denrées alimentaires (et parfois un groupe de consommateurs) sont en relation directe. Il leur arrive de conclure des contrats qui stipulent les quantités à livrer, les prix et les variétés.

101. Les producteurs de produits différenciés bénéficient d'une marge plus intéressante s'ils passent par le Sanchoku que s'ils empruntent les circuits commerciaux ordinaires. Bien entendu, les détaillants sont tributaires des marchés de gros, sur lesquels ils peuvent se procurer une quantité définie de produits tout au 
long de l'année, mais ils s'approvisionnent aussi auprès du Sanchoku pour différencier leurs produits et attirer des groupes spécifiques de consommateurs. L'enquête ALIC, conduite en 2003 auprès de 170 entreprises agricoles productrices de légumes, a montré que $40 \%$ des exploitations de l'échantillon étaient sous contrat et vendaient plus de $80 \%$ de leur production dans ce cadre.

\section{Années 1990 : Essor des marchés de producteurs}

102. Depuis les années 1990, les marchés de producteurs agricoles (points de vente directe) sont devenus un circuit de distribution alimentaire important. D'après le recensement agricole effectué par les pouvoirs publics en 2005, il en existe 13538 dans tout le Japon. Il ressort d'une autre enquête officielle que le chiffre d'affaires moyen de ces marchés est de 100 millions JPY par an et que certains d'entre eux engrangent même plus de 2 milliards JPY par an.

103. Selon une estimation réalisée par Kobayashi et al. (2008) pour le PRIMAFF, les consommateurs se sont procurés dernièrement environ $10 \%$ de leurs légumes frais sur les marchés de producteurs. Ces derniers permettent aussi aux agriculteurs d'accroître leurs marges au sein de la filière agroalimentaire. En effet, les exploitants présents sur les marchés de producteurs sont à même de déterminer les prix de leurs produits, alors que les prix des fruits et légumes commercialisés sur les marchés de gros sont généralement déterminés par voie d'enchères. De plus, ces agriculteurs ne sont pas tenus de respecter des normes particulières ou des impératifs de volume, contrairement à ce qui se passe dans le circuit de commercialisation classique. Selon les caractéristiques de leur production, les exploitants peuvent conjuguer différents circuits. Autre avantage important des marchés de producteurs pour les agriculteurs : ils les mettent en relation directe avec les consommateurs. En conséquence, ils peuvent s'adapter pour répondre à la demande des consommateurs ou l'anticiper.

\section{Le mouvement Chisan-chisho : promotion des produits locaux depuis la fin des années 1990}

104. A la fin des années 90, les pouvoirs publics japonais ont commencé à encourager activement le mouvement Chisan-chisho, qui vise à favoriser la consommation des produits alimentaires locaux. Le Plan agricole fondamental de 2005, qui organise l'application concrète de la loi fondamentale sur l'alimentation, l'agriculture et les zones rurales, stipule que les pouvoirs publics encouragent le mouvement Chisan-chisho pour promouvoir la production agricole et augmenter le degré d'autosuffisance. En 2008, les collectivités locales et les coopératives agricoles ont rédigé 997 plans d'action locaux pour soutenir le mouvement Chisan-chisho, y compris l'utilisation des aliments produits localement dans la restauration scolaire. Les pouvoirs publics apportent leur appui en subventionnant la construction de marchés de producteurs, en dispensant des formations techniques et en assurant des échanges d'informations entre régions.

\section{Développement de la filière d'exportation de produits différenciés}

105. Ces dernières années, les exportations de produits agricoles sont devenues une source de plus en plus importante de revenus pour les agriculteurs au Japon. Sous l'effet du développement économique rapide des pays d'Asie de l'Est, les exportations de produits agricoles, forestiers et halieutiques japonais ont progressé de $36 \%$ entre 2000 et 2006. Cette augmentation est particulièrement sensible dans le cas des produits différenciés, notamment des produits halieutiques, des fruits frais et du thé vert. Par exemple, les cultivateurs de la ville d'Obihiro, sur l'île d'Hokkaido, vendent du nagaimo (igname) au Taipei chinois depuis 1999. Ils ont constaté que les consommateurs du Taipei chinois préféraient les produits de grande taille (contrairement aux consommateurs japonais) et qu'ils étaient disposés à les payer plus cher. Depuis quelques années, la majeure partie des nagaimo produits à Obihiro est exportée, y compris aux États-Unis et à Singapour. 
106. Les pouvoirs publics ont commencé à encourager activement les exportations de produits agricoles. En mars 2005, ils ont annoncé un plan de promotion des exportations, qui vise à doubler la valeur de ces dernières d'ici à 2009, c'est-à-dire à la porter à 600 milliards JPY. Dans le cadre du Plan fondamental pour l'alimentation, l'agriculture et les zones rurales, le plan annuel 2007 fixe même des objectifs encore plus ambitieux qui visent à porter la valeur des exportations à 1000 milliards JPY d'ici à 2013 moyennant l'accélération des négociations sur la quarantaine avec les pays importateurs et le marketing à l'étranger.

\section{Apport des mouvements alternatifs de distribution aux producteurs : les marchés de producteurs}

107. Les produits agricoles vendus sur les marchés de producteurs sont en général moins chers que ceux qui sont distribués dans les commerces classiques, mais permettent aux agriculteurs d'augmenter leurs revenus nets car ils abaissent les coûts de commercialisation et réduisent les pertes à cause de normes moins restrictives.

108. Kobayashi et al. (2008) ont évalué les effets économiques des marchés de producteurs, en s'appuyant sur l'exemple du Magasin A, qui a enregistré un chiffre d'affaires de 2.49 milliards JPY en 2006. Ce point de vente se trouve dans la plaine agricole de la préfecture de Wakayama et compte environ 1400 fournisseurs. Le chiffre d'affaires par exploitation est de 1.17 million JPY par an. Au total, le magasin a reçu quelque 800000 clients venus d'une zone d'un rayon de $30 \mathrm{~km}$ qui comprend Osaka, deuxième ville du Japon. D'après les estimations, les producteurs affiliés gagnent en moyenne $38 \%$ de plus que s'ils passaient par les marchés de gros classiques et les clients font une économie de $16 \%$ par rapport aux consommateurs qui s'approvisionnent chez les détaillants ordinaires. De plus, le magasin emploie 5 salariés à temps complet et 48 à temps partiel.

109. Bien que les circuits classiques occupent une place prépondérante dans le commerce alimentaire au Japon, d'autres circuits se sont développés depuis quelques décennies pour répondre aux nouvelles préoccupations des consommateurs concernant, entre autres, la sécurité des aliments et la préservation de l'environnement. Les principaux atouts des circuits alternatifs tiennent au fait qu'ils réduisent les marges commerciales, mais aussi qu'ils permettent aux producteurs de répondre plus rapidement aux diverses attentes des consommateurs, compte tenu de la proximité qu'ils instaurent entre les uns et les autres. Par ailleurs, les producteurs ont une plus grande maîtrise des prix et accroissent leur marge à l'intérieur de la filière alimentaire. En général, l'agriculture sous contrat permet aux exploitants de partager les risques liés à la production avec les acheteurs. Cependant, il y a peu de chances pour que ces réseaux alternatifs remplacent les circuits de commercialisation habituels, fondés sur les marchés de gros, car ceux-ci présentent aussi des avantages : ils assurent un approvisionnement tout au long de l'année en produits alimentaires bon marché et de qualité constante, et ils jouissent d'une bonne réputation. Par exemple, les produits de l'agriculture biologique étaient auparavant commercialisés par les circuits alternatifs comme les Teikei, mais ils sont aussi très présents, désormais, sur les marchés de gros ordinaires. Les producteurs sont censés tirer le meilleur parti possible des réseaux de commercialisation des produits alimentaires en conjuguant les différents circuits selon les caractéristiques de leurs produits.

\section{A-II. Irrigation au goutte à goutte-fertirrigation et techniques nucléaires (Turquie)}

110. L'Agence Internationale de l'Énergie Atomique est vivement remerciée pour l'aide apportée dans la préparation de cette étude de cas.

\section{Le problème}

111. En 2006, la Turquie représentait $3 \%$ des exportations de pommes de terre fraîches, ce qui faisait d'elle le cinquième exportateur mondial. Près du tiers de la production totale de pommes de terre 
(approximativement 1.2 million de tonnes) provient de la région de Niğde-Nevshir. Cette zone étant caractérisée par un climat continental marqué (températures estivales moyennes de $24^{\circ} \mathrm{C}$ et températures hivernales moyennes de $-2^{\circ} \mathrm{C}$ ), les 2000 agriculteurs de la région, qui exploitent près de 30000 hectares, sont tributaires des eaux souterraines résiduelles apportées par les précipitations de printemps. Les pénuries d'eau sont donc un problème constant. Sous-division de l'Autorité Turque de l'Énergie Atomique (ATEA), le Centre de recherches nucléaires et de formation de Saraykoy, qui a pour objet la recherche scientifique et la formation des agronomes, mais aussi des agriculteurs confrontés à un risque de diminution de la productivité de leurs terres et de pollution de l'eau, a sollicité l'appui de l'Agence internationale de l'énergie atomique (AIEA).

112. Le sol sablo-limoneux de la région se caractérise par sa texture légère. Il en découle que des applications d'engrais azotés ou une irrigation excessives ou inappropriées le rendent très sujet à la détérioration, se traduisent par un gaspillage des ressources naturelles (eau) et entraînent une dégradation de la qualité de l'eau liée à l'infiltration des nitrates et au ruissellement de ceux-ci dans les eaux de surface et les eaux souterraines.

\section{Nouvelles méthodes}

113. Ces dix dernières années, les services agronomiques du ministère de l'Agriculture ont recherché des solutions plus adaptées, du point de vue technologique, aux différents problèmes rencontrés. L'Autorité Turque de l'Énergie Atomique (ATEA) a donc uni ses forces à celles des chercheurs de l'Institut de recherche sur la pomme de terre de Nigde et de l'Institut de recherche sur les sols et les engrais, dans le but d'améliorer la productivité des sols et de résoudre les problèmes environnementaux soulevés par l'activité agricole. Dans le cas qui nous occupe, l'ATEA a fait appel à l'AIEA. Ensuite, quelques exploitants, en collaboration avec des organismes de recherche gouvernementaux, universitaires ou autres, ont conjugué leurs efforts pour mettre en œuvre un système innovant d'irrigation et de fertilisation qui fait en partie appel à des techniques nucléaires.

\section{Description des projets}

114. L'ATEA-AIEA a lancé trois projets distincts dans l'optique de répondre aux besoins des agriculteurs de la région de Niğde-Nevshir. Le premier, conduit de 1992 à 1994, portait sur l'irrigation par aspersion (Halitligil et al., 2002) et les deux suivants, de 1997 à 2000 (Halitligil et al., 2003) puis de 2005 à 2008, sur l'irrigation au goutte à goutte-fertirrigation. Le projet mené de 1997 à 2000 visait à expérimenter l'efficacité de l'irrigation au goutte à goutte et de la fertirrigation dans la culture de la pomme de terre dans cette région précise. Le but était d'accroître les rendements au moyen de ces deux techniques nouvelles, en améliorant l'efficacité des apports d'azote et de l'irrigation, tout en réduisant la pollution par les nitrates. Le troisième projet, conduit de 2005 à 2008 sur la base du succès du premier, avait pour objectifs d'améliorer l'état des sols et d'accroître encore l'efficience de l'irrigation et l'absorption de l'azote par les tubercules, moyennant l'utilisation de la vesce comme engrais vert la deuxième année de la rotation pomme de terre-blé, ou de la luzerne comme engrais vert/culture fourragère dans un système luzerne-luzerne-pomme de terre sur trois ans.

\section{Innovation technique et test à grande échelle}

115. L'irrigation au goutte à goutte et la fertirrigation existent depuis un certain temps, mais l'innovation vient ici du fait que l'on a eu recours, en plus, à la technologie nucléaire pour quantifier l'amélioration de l'efficience des techniques d'irrigation appliquées à la pomme de terre. L'irrigation au goutte à goutte passe par un réseau de canalisations et de goutteurs qui apporte l'eau soit au niveau des racines, soit à la surface du sol, de manière à n'arroser que la plante et à limiter au minimum le volume d'eau apporté. La fertirrigation consiste à appliquer les engrais en les dissolvant dans l'eau distribuée par le 
réseau d'irrigation au goutte à goutte. Le premier projet a démarré avec trois agriculteurs de la région, sélectionnés en raison de leur aptitude à mettre en œuvre la nouvelle technologie et formés par les spécialistes de l'AIEA. Il a d'abord consisté à remplacer sur certaines parcelles un réseau d'irrigation classique par aspersion par un réseau d'irrigation au goutte à goutte-fertirrigation. Huit réseaux ont ainsi été installés la première année. La deuxième, l'un des agriculteurs a accepté d'étendre l'expérimentation de la fertirrigation à une superficie de $10000 \mathrm{~m}^{2}(1 \mathrm{ha})$.

116. Le deuxième projet faisait appel aux techniques nucléaires (sondes à neutrons et isotopes stables ${ }^{15} \mathrm{~N}$ ) dans l'optique d'améliorer encore l'efficience de l'irrigation au goutte à goutte et de la fertirrigation du point de vue de la gestion des sols, de l'eau et des éléments nutritifs durant la période de végétation. Les sondes à neutrons sont employées pour mesurer la quantité d'eau présente dans le sol. Une fois qu'elles sont enfoncées dans le sol et qu'une réaction nucléaire est déclenchée, des neutrons rapides sont produits et libérés. Ceux-ci entrent en collision avec les noyaux d'hydrogène (un composant de l'eau), ce qui les ralentit, et ils sont ensuite décomptés; le résultat de ce calcul permet d'estimer la quantité d'eau présente dans un échantillon de sol (AIEA, 2008). Ce procédé fournit aux cultivateurs des informations utiles sur le volume d'eau à apporter pour favoriser la croissance des végétaux. L'isotope stable de l'azote $\left({ }^{15} \mathrm{~N}\right)$ ajouté aux engrais azotés peut être utilisé comme marqueur du devenir de l'azote dans un système sol-plante. Après l'apport de l'engrais azoté marqué au ${ }^{15} \mathrm{~N}$, il est possible de procéder à des mesures sur les cultures pour évaluer l'efficience avec laquelle l'engrais est assimilé par les végétaux, d'une part, et la proportion qui s'est infiltrée dans le sol hors de portée des racines, d'autre part. Ces informations permettent une gestion plus précise des engrais azotés, que ce soit dans le temps, du point de vue des méthodes employées ou de la source.

117. Les cultivateurs et les chercheurs ont estimé que les techniques nucléaires recommandées par l'AIEA et l'ATEA aidaient nettement à améliorer la conduite des cultures dans son ensemble. Les premiers résultats du troisième projet indiquent que la vesce n'accroît pas autant que la luzerne la matière organique du sol, condition première pour améliorer la qualité du sol et donc l'efficience de l'utilisation des engrais et de l'eau sur les terres sablonneuses de la région de Niğde-Nevshir. Ici aussi, les sondes à neutrons employées pour mesurer l'humidité du sol et le ${ }^{15} \mathrm{~N}$ se sont révélés très utiles pour évaluer les incidences de l'emploi de la vesce et de la luzerne sur la consommation et la gestion des intrants tels que les engrais et l'eau d'irrigation. Dans la mesure où le cycle de rotation lancé sur le site d'expérimentation n'est pas encore arrivé à son terme, il faudra attendre 2009 pour obtenir des résultats plus précis. Les étapes suivantes de ce troisième projet devraient être particulièrement utiles aux agriculteurs de la région de Niğde-Nevshir dans le futur.

118. La Division mixte FAO/AIEA des techniques nucléaires dans l'alimentation et l'agriculture considère que l'utilisation des techniques nucléaires dans ce projet, comme dans d'autres, présente des avantages et a aussi ses limites. Pour de plus amples précisions à ce sujet, veuillez vous reporter au lien donné en note de bas de page ${ }^{21}$.

\section{Résultats}

119. Pour les agriculteurs qui ont adopté ces nouvelles méthodes, le coût d'installation d'un réseau d'irrigation au goutte à goutte-fertirrigation s'est monté à quelque 200 USD par hectare. De plus, la maintenance leur est facturée environ 30 USD par hectare pour une période de cinq ans.

120. L'abandon de l'irrigation par aspersion au profit de l'irrigation au goutte à goutte-fertirrigation a eu une incidence extrêmement positive sur la consommation d'eau et d'engrais. Les réseaux d'irrigation par aspersion entraînent des déperditions de près de $65 \%$, car toute l'eau ne parvient pas aux racines des

21 http://www-naweb.iaea.org/nafa/swmn/use-nuclear-tech.pdf. 
plants de pomme de terre. Il en va de même des applications d'engrais du fait qu'une grande partie de l'eau n'est pas accessible aux racines. Contrairement à l'irrigation par aspersion et à l'épandage en pleine surface des engrais azotés, l'irrigation au goutte à goutte-fertirrigation permet de canaliser l'eau et l'engrais précisément vers le système racinaire et se traduit ainsi par une diminution de $50 \%$ de la consommation d'eau d'irrigation. En ce qui concerne les engrais azotés, les cultivateurs ont vu leur consommation passer de $1000 \mathrm{~kg} \mathrm{~N} / \mathrm{ha}$ à $600 \mathrm{~kg} \mathrm{~N} / \mathrm{ha}$ sans baisse de rendement. En outre, la diminution de la consommation d'engrais azoté limite le risque de pollution par les nitrates (Halitligil et al., 2002). Ainsi, l'irrigation au goutte à goutte-fertirrigation réduit les coûts imputables aux intrants et limite au minimum les risques de détérioration des sols, sans porter atteinte aux rendements. De plus, les cultivateurs font une économie de temps, d'énergie et de travail dont la valeur est estimée à 2000 USD par hectare. Si l'irrigation au goutte à goutte-fertirrigation était appliquée à toute la région, les économies annuelles pourraient se monter au total à près de 60 millions USD, ce qui se traduirait par une augmentation considérable de la rentabilité.

121. L'ATEA-AIEA a mis en place des séminaires et des formations tous les ans pour améliorer les performances et organiser les cultivateurs et chercheurs participants en réseau.

122. En novembre 2004, les pouvoirs publics ont pris acte de l'utilité économique et environnementale de l'irrigation au goutte à goutte-fertirrigation, et offert des prêts à faible taux d'intérêt aux exploitants de la région disposés à adopter cette pratique. En trois ans seulement, les superficies équipées dans la région de Niğde-Nevshir sont passées de 500 à 4000 hectares. Fin 2007, le ministère de l'Agriculture a commencé à subventionner à hauteur de $50 \%$ les dépenses d'irrigation au goutte à goutte dans toute la Turquie ; pour bénéficier des aides, les exploitants doivent produire un rapport technique détaillant leurs dépenses, établi par l'un des experts spécialement habilités à cette fin. Il en découle que, dans les années à venir, davantage de cultivateurs pratiqueront l'irrigation au goutte à goutte-fertirrigation, notamment en Cappadoce.

123. Pour de plus amples renseignements sur l'irrigation au goutte à goutte-fertirrigation et les techniques nucléaires évoquées dans la présente étude de cas, veuillez consulter M. B. Halitligil (2007), 'Drip Irrigation and Fertigation: Turkey', Soils Newsletter Vol. 30, No.1 : 9-10.

\section{A-III. Pôle de compétitivité Industries et Agro-Ressources (France)}

124. L'assistance de Mr. Duchâteau (Pôle de compétitivité Industries et Agro-Ressources), de Mr Dutartre (Président d'A.R.D.), de Mr Le Hénaff (Directeur Géneral d'A.R.D.) et de Mr Prévoteau (Président de Soliance) a été précieuse dans la rédaction de cette étude de cas.

\section{La coopération au sein du secteur agricole dans le Nord Est de la France}

125. La tradition de coopération entre producteurs agricoles du Nord-est de la France est établie depuis plus d'un siècle. Historiquement, les producteurs cherchaient à se protéger des risques liés à un environnement économique difficile. Le Nord-est de la France était en effet une région isolée, éloignée des ports et relativement pauvre. Dès la première moitié du vingtième siècle, le développement de coopératives de taille importante pouvant peser sur les marchés est amorcé.

126. Profitant des conditions économiques stables favorisées par la Politique Agricole Commune (PAC), la coopération entre producteurs agricoles de grandes cultures s'est progressivement renforcée. Au gré de regroupements et de fusions, elle a évolué pour englober des activités de transformation (malteries, meunerie, sucrerie, amidonnerie). Les régions Picardie et Champagne-Ardenne sont aujourd'hui des leaders français dans la production de céréales, d'oléagineux, de betteraves à sucre et de pommes de terre.

127. Ainsi, le Nord-est de la France, mondialement connu pour sa production de Champagne, abrite aussi des champions des grandes cultures avec, pour l'industrie sucrière, Cristal Union et, pour l'industrie 
céréalière, le premier groupe céréalier européen: la coopérative Champagne Céréales. Cette coopérative regroupe près de 8800 exploitants, collecte 2.5 millions de tonnes de céréales par an et a un chiffre d'affaire annuel de 1.3 milliards d'euros.

128. Champagne-Céréales est bien plus qu'une coopérative permettant à ses membres de diminuer leurs coûts de production en partageant matériels et achats d'intrants ou encore d'atteindre une taille critique pour accéder à certains marchés. C'est un véritable groupe industriel à dimension internationale avec une structure financière complexe. Par l'intermédiaire de filiales, ce groupe est impliqué dans les activités en amont et en aval de la production agricole: la distribution d'engrais, la transformation des produits, la commercialisation et même des activités de recherche et développement.

\section{Valorisation non alimentaire : une des voies choisies pour créer de la valeur}

129. Pour les acteurs principaux du secteur agricole dans le Nord-est de la France, la création de valeur passe par la valorisation alimentaire et non-alimentaire des produits végétaux. Ces deux approches complémentaires s'inscrivent dans le développement de procédés de raffinerie végétale, c'est-à-dire dans le développement de procédés permettant une utilisation des matières premières végétales dans leur ensemble pour produire de la nourriture, de l'énergie et tout un ensemble de bioproduits.

130. Le fer de lance de la recherche privée sur la chimie du végétal en Champagne Ardenne est la société Agro Industrie Recherches et Développements (A.R.D.). Cette société a été créée en 1989 lorsque Champagne Céréales et Sucre Union, la filiale commerciale des coopératives sucrières Cristal Union et Sucrerie de Bourdon, ont décidé de fusionner leurs programmes de recherche Ethanol Recherches et Développements (E.R.D.) et Sucre Recherches et Développements (S.R.D.). Le développement des activités d'A.R.D. s'est accéléré avec la réforme de la PAC. En 1994, A.R.D. crée Soliance, sa filiale en matière de production et de commercialisation de produits cosmétiques issus de la chimie du végétal en particulier de tensio-actifs. Actuellement, A.R.D. et Soliance, dont les activités de développement et de production sont basées sur le site agro-industriel de Pomacle dans la Marne, comptent 125 employés à majorité qualifiés ou hautement qualifiés.

131. Les motivations des coopératives de grande culture pour investir dans la R\&D sont de développer de nouveaux produits et procédés issus de la chimie du végétal permettant d'optimiser la valorisation de la production et les procédés de transformation. Les projets menés par A.R.D. touchent au raffinage du végétal, à la chimie verte et aux biotechnologies blanches. L'objectif d'ARD est de développer des réponses techniques économiquement viables aux problématiques liées à la chimie du végétal. Les projets comprennent différentes étapes successives : la recherche fondamentale, la recherche appliquée, le projet pilote et ensuite le projet industriel. La recherche fondamentale est souvent conduite en partenariat avec la recherche publique, les brevets sont donc déposés en copropriété. Actuellement A.R.D. dépose de 2 à 3 brevets par an.

132. L'actionnariat d'A.R.D. représente uniquement $30 \%$ des frais de fonctionnement de l'entreprise de R\&D. Il est constitué des coopératives céréalières à plus de $50 \%$, des coopératives sucrières (près de $18 \%$ ), des coopératives luzernières (près de 5\%), du Crédit Agricole du Nord-est (près de 16\%) et de l'amidonnerie Chamtor (près de $8 \%$ ), elle-même filiale de Champagne Céréales. A titre d'exemple, les producteurs de Champagne Céréales reversent 0.15 euros par tonne de céréales au financement de la recherche. $70 \%$ des frais de fonctionnement sont donc assurés par des sources extérieures : les revenus des brevets, le mécanisme d'intégration fiscale avec Soliance et les financements publics (Crédit Impôt Recherche, programmes subventionnés au niveau national, régional ou européen). 


\section{Le pôle de compétitivité renforce la mise au point de projets innovants}

133. Le nombre de projets innovants auxquels A.R.D. est associé a connu une accélération importante avec la mise en place du pôle de compétitivité à vocation mondiale Industries et Agro-Ressources (IAR) le 12 Juillet 2005. Le pôle IAR fait partie des 71 pôles qui ont été labellisés par le gouvernement français ${ }^{22}$. Ces pôles (ou «clusters ») touchent à tous les domaines de l'économie et se définissent comme la combinaison, sur un espace géographiquement donné, d'entreprises, de centres de formation et d'unités de recherche, engagés dans une démarche partenariale destinée à dégager des synergies autour de projets communs à caractère innovant, et disposant de la masse critique nécessaire pour une visibilité internationale.

134. L'ambition du pôle IAR est de devenir le leader européen de l'innovation dans les transformations non alimentaires des agro-ressources et de la biomasse. Il se structure autour d'une agriculture performante, établie et organisée, d'un réseau d'acteurs efficaces et reconnus dans le domaine de la recherche publique et privée et de la présence de groupes industriels importants dans le domaine de la transformation de produits agricoles (Sucre Union et Champagne Céréales par exemple). Le pôle se base sur une masse salariale de quelques 26200 personnes dont près de 1000 emplois dans le domaine de la recherche. Il est organisé grâce à une association qui sert d'intermédiaire entre les financeurs de projets innovants (comme l'agence pour l'innovation Oséo, Agence Nationale de la Recherche, les instances européennes ou régionales) et les acteurs du pôle qui collaborent ensemble sur ces projets. Cette association a aussi une mission de veille économique et d'accompagnement dans le développement de la notoriété.

135. Un des projets majeurs porté par le pôle IAR est le projet Futurol ${ }^{23}$ qui a été lancé le 11 Septembre 2008. Le montant global de ce projet s'élève à 74 millions d'euros sur la période 2008-2016 dont 30 millions financés par Oséo. Il associe 11 acteurs scientifiques, industriels et financiers au sein du consortium Procethol 2G: A.R.D., la Confédération Générale des Betteraviers, Champagne Céréales, le Crédit Agricole du Nord Est, l'Institut Français du Pétrole, 1'Institut National de la Recherche Agronomique, Lesaffre, l'Office National des Forêts, Tereos, Total et Unigrains et vise le développement et la commercialisation d'un procédé complet de production de bioéthanol de 2ème génération à partir de plantes entières ou de biomasse lignocellulosique. Les retombées potentielles pour les différents acteurs impliqués sont importantes. Le projet comporte une phase pilote, suivie d'une phase prototype. L'installation pilote sera construite sur le site agro-industriel de Pomacle dans la Marne, site où sont déjà implantées A.R.D. et les différentes entreprises qui participent à la raffinerie du végétal.

136. Le pôle IAR assoit les connaissances et les compétences développées depuis longtemps par les acteurs du secteur dans le Nord-est de la France. Il leur permet d'étoffer et de renforcer les projets innovants développés et d'envisager des retombées économiques importantes pour la région, avec par exemple la création d'emplois autour des usines de raffinerie végétale et de biocarburants de seconde génération.

137. Le pôle facilite aussi la reconnaissance internationale et la mise en place de partenariats avec des entreprises innovantes hors de France. Bien entendu, il repose sur la présence d'entreprises «leaders »

22

Après le dépôt d'un acte de candidature qui demandait la mise au point d'un projet de collaboration entre monde de la recherche et monde industriel, les pôles ont été sélectionnés par l'état français sur leur capacité à renforcer la compétitivité de la France, à dynamiser le développement économique et à favoriser la création d'emplois.

23 、 Le dossier de presse du projet Futurol est disponible à l'adresse suivante :

http://www.inra.fr/content/download/14001/172255/version/1/file/DP-Futurol-final.pdf 
dans la région et sur une longue tradition de coopération. Ce cluster n'est pas directement transposable pour d'autres régions au sein de pays de l'OCDE mais permet d'envisager les opportunités potentielles créées par le développement de la bioéconomie dont peuvent bénéficier les acteurs du secteur agroalimentaire et les producteurs agricoles en particulier.

\section{A-IV. Quelques exemples d'aliments fonctionnels enrichis en oméga-3 (France, Canada, Allemagne)}

138. En près de trente ans, les acides gras polyinsaturés ont pris une place prédominante sur le marché des aliments fonctionnels. De nombreuses études montrent que les oméga-3 DHA (un type d'acide gras polyinsaturé) jouent un rôle important dans l'entretien de la santé et qu'ils présentent un potentiel de prévention/régulation des maladies. Ces substances se révèlent capables d'avoir des effets bénéfiques sur la santé, comme la diminution du cholestérol, l'augmentation de la teneur du sang en acide docosahexaénoïque (DHA), qui accroîtrait le flux sanguin vers le cœur, ou d'autres avantages cardiovasculaires $^{24}$. Elles sont donc susceptibles de contribuer à faire diminuer le risque de maladie cardiovasculaire et à lutter contre les affections inflammatoires comme l'arthrite, entre autres (Filmer, 2006 ; Kris-Etherton et al., 2002 ; Weststrate et al., 2002).

139. L'organisme ne sachant pas synthétiser les oméga-3, ceux-ci doivent être apportés par l'alimentation. La principale source d'oméga-3 DHA est habituellement l'huile de poisson, notamment de poissons gras comme le saumon, la sardine et le maquereau. Pour s'assurer des apports suffisants, de plus en plus de gens consomment des aliments enrichis en oméga-3. Des entreprises du monde entier recherchent donc des méthodes innovantes pour mettre au point ce type d'aliments fonctionnels.

140. Ci-dessous sont présentés trois exemples de développement d'aliments fonctionnels impliquant directement des producteurs agricoles ainsi que d'autres acteurs de la chaîne. Ces trois exemples ont pour caractéristiques communes de miser sur les produits enrichis en oméga-3. Abstraction faite de l'innovation technologique, chacune des entreprises présentées dans cette étude de cas répartit les risques commerciaux en s'adossant à une organisation de poids, qu'il s'agisse d'une coopérative forte de 6000 éleveurs laitiers comme Humana ou d'une association rassemblant les acteurs présents à toutes les étapes de la filière comme l'association Bleu-Blanc-Coeur. La précision avec laquelle la production est conduite grâce à ces formes d'organisation semble nécessaire pour assurer une certaine teneur en éléments nutritifs spéciaux.

\section{Les acides gras polyinsaturés oméga-3 présents dans les graines oléagineuses : Bleu-Blanc-Cour}

141. Fondée en août 2000 pour promouvoir une alimentation humaine saine à partir de techniques agricoles respectueuses de l'environnement, l'association Bleu-Blanc-Cœur produit plusieurs produits aux qualités nutritionnelles améliorées moyennant l'utilisation d'oléagineux (en particulier des graines de lin) dans la composition des aliments du bétail ou des aliments destinés à la consommation humaine. Elle est composée d'opérateurs spécialisés de la filière, des semenciers aux exploitants en passant par les fabricants d'aliments du bétail, les éleveurs et les entreprises de transformation de denrées alimentaires. Avec des chercheurs et même des consommateurs, ceux-ci forment un réseau de 300 agents. Les produits vont du pain à la farine, en passant par toutes sortes de produits animaux comme la viande de volaille, la viande porcine et bovine et les produits laitiers ; tous sont riches en oméga-3. Il est possible de se les procurer dans le monde sous différentes marques, qui sont souvent des sous-marques de marques nationales ou de marques de détaillants. Pour maintenir le niveau de qualité et d'authenticité, Bleu-Blanc-Cœur coordonne la filière de production. L'aventure des oméga-3 a démarré en 1993, lorsque Valorex, fabricant d'aliments du bétail en Bretagne (France), a incorporé des graines de lin cuites dans ses rations en raison de leurs caractéristiques zootechniques. Les recherches menées par Valorex et des organismes publics de recherche

24 Pour de plus amples renseignements, voir le site de l'International Food Information Council : http://www.ific.org/publications/factsheets/omega3fs.cfm. 
ont ensuite montré que l'utilisation des graines de lin dans l'alimentation du bétail avait un effet sur le profil des acides gras dans le sang des consommateurs de produits animaux (Weill et al, 2002). Il convient de noter que les graines oléagineuses riches en oméga-3 peuvent être substituées à l'herbe de printemps, particulièrement saine, et être employées tout au long de l'année. Grâce à un procédé d'extraction spécial mis au point par Valorex, les éléments nutritifs de ces graines peuvent être assimilés par les animaux. Les graines sont donc incorporées dans les rations consommées par les animaux dans la filière de production alimentaire Bleu-Blanc-Cœur. A l'heure actuelle, les graines Bleu-Blanc-Cœur (principalement des graines de lin) ne sont produites qu'en France, bien que les produits soient commercialisés à l'étranger, ce qui stimule l'activité économique dans les régions où sont pratiquées les cultures en question ${ }^{25}$.

\section{Acides gras polyinsaturés oméga-3 dans la viande : Les Petites Fermes du Centaure Inc.}

142. Moyennant une alimentation stricte des animaux d'élevage à partir de végétaux riches en acides gras oméga-3, la société Les Petites Fermes du Centaure est spécialisée dans les produits à base de viande à valeur ajoutée. En 2004, cette entreprise canadienne regroupant plusieurs petites exploitations familiales a été la première du pays à être autorisée à commercialiser de la viande porcine haut de gamme. Il a été démontré que l'ajout de sources d'acide alpha-linolénique comme les graines de lin dans les aliments du bétail augmentait la teneur en acides gras oméga-3 chez le porc et dans les produits à base de porc. Sous la marque VITO, Les Petites Fermes du Centaure produisent plusieurs produits alimentaires, des pepperoni aux saucisses, qui constituent tous une bonne «source d'acides gras polyinsaturés oméga- $3{ }^{26}$. L'utilisation de végétaux dans l'alimentation des animaux présente l'avantage de modifier totalement le profil des acides gras dans la viande de porc, car elle diminue la teneur en acides gras saturés et fait baisser le ratio oméga-6 sur oméga-3. D'après Santé Canada, le porc enrichi en oméga-3 contient deux fois plus d'acide alpha-linolénique que le porc ordinaire. Les Petites Fermes du Centaure se préoccupent non seulement du bien-être des consommateurs, mais aussi de l'environnement. Tous les exploitants participants doivent se conformer à un cahier des charges qui leur impose notamment de respecter le «plan agro-environnemental de fertilisation » conçu par les autorités locales. Ce plan assure que les pratiques agricoles sont saines et favorables à la préservation de l'environnement. Après plusieurs années de commercialisation, Les Petites Fermes du Centaure ont arrêté leur production. Le marché est porteur et les pouvoirs publics avaient donné leur feu vert, mais l'entreprise a été amenée à prendre cette décision à cause de plusieurs facteurs, notamment le manque de moyens pour commercialiser les produits, ainsi que d'autres problèmes non divulgués. A l'heure actuelle, elle est à la recherche de nouveaux partenaires pour redémarrer sa production.

\section{Acides gras polyinsaturés oméga-3 dans les produits laitiers : Humana GmbH}

143. Humana Milchunion eG, coopérative laitière classique regroupant plus de 6000 exploitations laitières, a constaté une nouvelle demande pour les aliments fonctionnels sur le marché des aliments pour bébés. Implantée à Herford, en Allemagne, Humana $\mathrm{GmbH}$, filiale de Humana Milchunion eG, produit une large gamme de produits laitiers vendus dans plus de trente pays. Depuis plus de 50 ans, Humana GmbH améliore les caractéristiques et la qualité de ses aliments pour bébé. Elle attribue aux acides gras polyinsaturés à longue chaîne oméga-3 un intérêt nutritionnel utile au développement de l'enfant. D'après certaines études, il pourrait exister un lien entre les acides gras oméga-3 et l'amélioration du développement du cerveau et de l'œil chez l'enfant. Dans sa série en cinq étapes, Humana GmbH a modifié certaines formules pour obtenir une teneur plus élevée en oméga-3 et en fibres alimentaires prébiotiques, dans le but de renforcer l'appareil digestif. La formule standard (étape 1) est obtenue avec

25 Pour de plus amples informations, voir le site web de Bleu-Blanc-Cœur : www.bleu-blanc-coeur.com. . 
des constituants du lait et des huiles végétales de qualité supérieure, lesquelles apportent les acides gras polyinsaturés à longue chaîne. Les produits Humana GmbH Baby Fit-2 et Humana Follow-on Formula 3 (étapes 2 et 3) apportent des fibres alimentaires prébiotiques. Humana GmbH garantit la qualité élevée de chacun de ses produits grâce à ses liens avec la coopérative laitière Humana Milchunion. Cette dernière permet de veiller à la traçabilité des produits et à l'assurance qualité. Le succès d'Humana s'étend à toute l'Allemagne, où cette entreprise occupe une position de leader dans de nombreux créneaux, avec ses aliments thérapeutiques pour bébés, des céréales instantanées hypoallergéniques pour bébés et des aliments pour bébés sans lait ${ }^{27}$.

\section{A-V. Des oléagineux enrichis en oméga-3 pour la durabilité et la santé (Australie)}

144. L'assistance de Mr Lee, Directeur du Programme Phare sur le Futur des Aliments (Food Futures Flagship Programme) au CSIRO (Commonwealth Scientific and Industrial Research Organisation), a été appréciée pour la rédaction de cette étude de cas

145. Innover dans la production d'aliments destinés à la consommation humaine et animale pour améliorer la santé et œuvrer à la durabilité écologique est l'un des principaux domaines de recherche du Programme Phare sur le Futur des Aliments du CSIRO. L'un des projets de recherche les plus prometteurs vise à développer des graines oléagineuses contenant des oméga-3 DHA.

\section{Durabilité de la filière halieutique}

146. La hausse de la demande de poisson et d'huile de poisson fait peser une pression de plus en plus forte sur les ressources halieutiques. La demande de poissons gras tels que le saumon, la sardine et le maquereau, tous riches en oméga- $3^{28}$, est en augmentation. L'élevage de certaines espèces de poissons atténue la pression exercée par la pêche sur les stocks naturels, mais il nécessite des huiles et des farines de poisson, ce qui présage une érosion de la viabilité à long terme de l'activité. Dernièrement, certains élevages ont essayé de substituer des huiles végétales aux huiles de poisson dans l'alimentation, ce qui amène à se demander si les poissons élevés aux huiles végétales sont aussi riches en oméga-3 DHA que les poissons nourris aux huiles de poisson. D'après certaines études scientifiques, la chair des poissons nourris uniquement avec des huiles végétales ne présente pas la même teneur en oméga-3 DHA (Bell et al., 2001, 2003, 2004 ; Mourente et Bell, 2006). Or, du point de vue de la santé, si le poisson contient moins d'oméga-3, il risque de paraître moins intéressant aux consommateurs.

\section{Mise au point d'oléagineux enrichis en oméga-3}

147. Le problème pourrait être résolu en trouvant un moyen d'incorporer des oméga-3 DHA dans les huiles végétales utilisées pour nourrir les poissons d'élevage. Les oméga-3 DHA sont produits principalement par des microalgues. Les poissons les ingèrent directement en consommant ces dernières ou indirectement en mangeant d'autres poissons. Compte tenu des effets bénéfiques que les oméga-3 peuvent avoir sur la santé, accroître leur disponibilité et assurer la durabilité de leur production présente un intérêt considérable pour le secteur, l'environnement et le consommateur.

148. L'objectif de l'équipe de recherche pluridisciplinaire du CSIRO était de mettre au point des plantes oléagineuses portant le gène des microalgues, de manière à obtenir des graines enrichies en

27 Pour de plus amples informations, voir le site web d'Humana : http://www.humana.de/en/.

28 Voir la $4^{\text {ème }}$ étude de cas au sujet des effets des oméga-3 DHA sur la santé et du développement d'aliments enrichis en oméga-3. 
oméga-3 DHA. A l'aide des techniques du génie génétique, les chercheurs sont parvenus à transférer le gène responsable de la production des oméga-3 DHA dans des plantes oléagineuses. Ils ont ainsi réussi à créer des graines de lin et des graines de colza enrichies en oméga-3 DHA, qu'ils ont utilisées dans les rations données à des poissons d'élevage. Il s'est avéré que la chair de ces poissons présentait une teneur en oméga-3 DHA supérieure à celle des poissons nourris uniquement avec des huiles végétales. En fait, la teneur en oméga-3 DHA était en l'occurrence équivalente à celle que l'on constate chez les poissons élevés avec des huiles de poisson.

\section{Nouveaux organismes génétiquement modifiés et acceptation par les consommateurs}

149. La mise au point de ces graines oléagineuses enrichies fait appel au génie génétique, ce qui soulève souvent des questions quant aux effets sur la santé humaine et sur l'environnement. Le CSIRO en est conscient et coopère avec l'organisme chargé de la réglementation des produits alimentaires (Food Standards Australia New Zealand, FSANZ) ${ }^{29}$. Que ces graines soient homologuées ou non, leur acceptation par les consommateurs ne va pas de soi. Cependant, si les consommateurs ont le sentiment qu'elles ont des effets bénéfiques sur la santé et que, de surcroît, elles contribuent à assurer la pérennité des ressources halieutiques mondiales, il y a plus de chances pour qu'ils acceptent l'utilisation de cette technologie.

\section{Quelles perspectives pour la filière halieutique?}

150. Une telle innovation a de multiples implications économiques. Dans le secteur agricole, les graines oléagineuses à valeur ajoutée offrent aux exploitants australiens un nouveau débouché dans le domaine des aliments utilisés en aquaculture et en agriculture ; de plus, les agriculteurs australiens peuvent aussi fournir des matières premières, en aval, au secteur alimentaire. Les producteurs de produits transformés qui utilisent des matières premières à valeur ajoutée peuvent améliorer leur produit en augmentant leur valeur nutritionnelle et créer de nouvelles niches. Le secteur de l'aquaculture tirera profit de l'utilisation des graines oléagineuses enrichies en oméga-3 dans l'alimentation des poissons moyennant l'augmentation des disponibilités et parce que l'on considérera qu'il contribue à éviter l'épuisement des stocks halieutiques mondiaux. A l'avenir, cette innovation devrait accroître la valeur des marchés agricoles de l'Australie. Le CSIRO prévoit que la commercialisation des graines oléagineuses enrichies en oméga-3 sera effective en 2013 au plus tôt.

29 La législation australienne et néo-zélandaise interdit la vente d'aliments génétiquement modifiés sans un étiquetage indiquant clairement la teneur en OGM. 


\section{RÉFÉRENCES}

Agriculture and Agri-Food Canada (2002), Potential Benefits of Functional Foods and Nutraceuticals to the Agri-Food Industry in Canada, Scott Wolfe Management Inc.

ALIC (2003), Report on contract farming in FY 2003 (in Japanese), Agricultural and Livestock Industries Corporation, Tokyo.

Baptista and P. Swann (1998), 'Do firms in clusters innovate more', Research policy, 27: 525-540.

Becattini, G. (1987), Mercato e forze locali: il distretto industriale, Bologna: II Mulino.

Bell, J. G. , J. McEvoy, D. R. Tocher, F. McGhee, P. J. Campbell et J.R. Sargent (2001), 'Replacement of Fish Oil with Rapeseed Oil in Diets of Atlantic Salmon Affects Tissue Lipid Compositions and Hepatocyte Fatty Acid Metabolism', Journal of Nutrition 131: 1535-1543.

Bell, J. G., R. J. Henderson, D. R. Tocher, J. Henderson, J. R. Dick and V. O. Crampton (2003) “Altered Fatty Acid Compositions in Atlantic salmon fed diets containing linseed and rapeseed oils can be partially restored by a subsequent fish oil finishing diet" Journal of Nutrition, 133, 9:2793-2801.

Bell, J. G., R. J. Henderson, D. R. Tocher et J. R. Sargent (2004), 'Replacement of Dietary Fish oil with increasing levels of linseed oil: Modification of Flesh Fatty Acid Composition in Atlantic Salmon Using Fish Oil Finishing Diet', Lipids, 39, 3: 223-32.

Blandford, D. (2000), 'The Functioning of Agriculture and Agribusiness in Developed Market Economies', In Agricultural Policy and the Enlargement of the European Union, A. Burrell et A. Oskam (eds), Wageningen Pers, Wageningen, Pays-Bas.

Coakley, M., E. Barrett, R. P. Ross, R. Devery et C. Stanton (2007), 'Cheese manufacture with milk with elevated conjugated linoleic acid levels caused by dietary manipulation', Journal of Dairy Science 90: 2919-2927.

Cook, M.L. et B. Plunkett (2006), 'Collective Entrepreneurship: An Emerging Phenomenon in ProducerOwned Organization', Journal of Agricultural and applied Economics 38, 2: 421-428.

Chaddad, F. R. et M. L. Cook (2004), 'Understanding New Cooperative Models: An Ownership-Control Rights Typology’, Review of Agricultural Economics 26,3: 348-360.

Crespi, J.M et S. Marette (2003), 'Some Economic Implications of Public Labeling', Journal of Food Distribution Research 34, 3: 83-94.

Dosi, G. (1982), 'Technological paradigms and technological trajectories', Research Policy 11, 2:147-62.

Dosi, G. (1988), 'Sources, procedures, and microeconomic effects of innovation'. Journal of Economic Literature 26 (1988), pp. 1120-1171.

DuPuis, E.M. (2006), 'Civic Markets: Alternative Value Chain Governance as Civic Engagement', Crop Management, doi: 10.1094/CM-2006-0921-09-RV. 
DuPuis, E.M., D. Goodman (2005), 'Should we go "home"' to eat?: toward a reflexive politics of localism', Journal of Rural Studies $21: 359-371$.

Filmer, M. (2006), ‘Omega-3 Crop quest within reach’, Farming Ahead 179: decembre 2006.

Fountas, S., S. M. Pedersen, S. Blackmore (2005), 'ICT in Precision Agriculture - diffusion of technology', In ICT in agriculture: perspective of technological innovation, E. Gelb and A. Offer (eds), http://departments.agri.huji.ac.il/economics/gelb-main.html .

Freeman, C. et F. Louca (2001), As Time Goes By: From the Industrial Revolution to the information Revolution, Oxford, Oxford University Press.

Frewer, L., J. Scholderer et N. Lambert (2003), 'Consumer acceptance of functional foods: issues for the future', British Food Journal 1005, 10: 714-731.

Giannakas, k. et A. Yiannaka (2008), 'Market and Welfare Effects of Second-Generation, ConsumerOriented GM Products', American Journal of Agricultural Economics 90, 1: 152-171.

Goldsmith, P. et H. Gow (2005), 'Strategic Positioning Under Agricultural Structural Change: A Critique of Long Jump Co-operative Ventures', International Food and Agribusiness Management Review 8, 2: $1-21$.

Gow, H. R., L. D. Oliver et N. G. Gow (2002), 'Co-operating to Compete in High Velocity Global Markets: The Strategic Role of Flexible Supply Chain Architectures', Journal on Chain and Network Science 2, 1: 19-32.

Gow, H. R., L. D. Oliver et N. G. Gow (2003), 'Value Creation in Farmer-Driven Marketing channels: the Case of Murrellen Pork', Journal of Food Distribution Research 34, 1: 86-91.

Gray, A. W. et M. D. Boehlje (2007), 'The Industrialization of Agriculture: Implications for future Policy', Purdue University, working paper 07-10.

Halitligil, M. B., A. Akin et A. İlbeyi (2002), 'Nitrogen balance of nitrogen-15 applied as ammonium sulphate to irrigated potatoes in sandy textured soils', Biology and Fertility of Soils 35 :369-378.

Halitligil, M. B., H. Onaran, N. Munsuz, H. Kislal, A. Akin, A. L. Unlenen, G. Cayc1 et C. Kutuk (2003), 'Drip irrigation and Fertigation of Potato under Light-textured soils of Cappadocia Region', Page 219-224, In Environmental Protection against Radioactive Pollution, N. Birsen and K. K. Kadyrzhanov (eds) Kluwer Academic Publishers. Imprimer aux Pays-Bas.

Hanf, J.H. et A. Pieniadz, 'Chain quality management in co-operatives', Conference Paper, 47th Annual Conference, German Association of Agricultural Economists (GEWISOLA),

Freising/Weihenstephan, Allemagne, septembre 26-28, 2007.

IAEA (2008), 'Field Estimation of Soil Water Content: A Practical Guide to Methods, Instrumentation, and Sensor Technology'. IAEA-TCS-30. International Atomic Energy Agency, Vienne, Autriche. ISSN 1018-5518. p. 123-129.

Kris-Etherton, P. M., W. S. Harris, L. J. Appel (2002), 'Fish Consumption, Fish Oil, Omega-3 Fatty Acids, and Cardiovascular Disease', Circulation 106: 2747-2757. 
Krugman, P. (1991), "Increasing returns and geographic locations", Journal of Political economy 99 3:483499

Kobayashi, K., Sato et Oohashi (2008), 'A Study on the Economic Effects of "Local Production for Local Consumption" - Centring on the Economic Effects the Farmers Markets have on Producers and Consumers-', Policy Research Institute, Ministry of Agriculture, Forestry and Fisheries in Japan, Tokyo.

Labrecque, J., M. Doyon, F. Bellavance et J. Kolodinsky (2006), 'Acceptance of Functional Foods: A Comparison of French, American, and French Canadian Consumers', Canadian Journal of Agricultural Economics 54: 647-661.

Lawson, R., J. Guthrie, A. Cameron, W. Fischer (2008), 'Creating value through cooperation: an investigation of farmers' markets in New Zealand', British Food Journal 110, 1: 11-25.

Lazzarini, S. G., F. R. Chaddad et M. L. Cook (2001), 'Integrating supply chain and network analyses: The study of netchains', Chain and network science 1, 1: 7-22.

Lindgreen, A. et F. Wynstra (2005), 'Value in business markets: What do we know? Where are we going?' Industrial Marketing Management 34, 732-748.

Linnemann, A. R., M. Benner, R. Verkerk et M. A.J.S. van Boekel (2006), 'Consumer-driven food product development', Trends in Food Science and Technology 17, 184-190.

Loureiro, M.L. et J.J. McCluskey (2000), 'Assessing Consumer Response to Protected Geographical Identification Labeling’, Agribusiness 16, 3: 309-320.

McCluskey J.J. et Loureiro, M.L. (2003), 'Consumer Preferences and Willingness to Pay for Food Labeling: A Discussion of Empirical Studies', Journal of Food Distribution Research 34, 3: 95-102.

Ménard, C. (2007), 'Cooperatives: hierarchies or hybrids'. Page 1-17. In Vertical Markets and Cooperatives Hierarchies, K. Karantininis and J. Nilsson (eds) Springer. Imprimer aux Pays-Bas.

Mourente, G. et J. G. Bell (2006) "Partial replacement of dietary fish oil with blends of vegetable oils in diets for European sea bass over a long term growth study: effects on muscle and liver fatty acid composition and effectiveness of a fish oil finishing diet”,Comp Biochem Physiol B.Biochem Mol Biol, 145, 34:389-399.

Murdoch, J. (2000), 'Networks - a new paradigm of rural development', Journal of Rural Studies 16, 407419.

Nelson R. (1993), National innovation Sytems: A comparative analysis New York, Oxford University press.

Nelson R. et N. Rosenberg N. (1994), 'American universities and technical advance in industry', Research Policy 23, 323-348.

OCDE (2000), Appellations d'origine et indications géographiques dans les pays membres de l'OCDE, OCDE, Paris. 
OCDE (2006), The Bioeconomy to 2030: Designing a Policy Agenda, OCDE, Paris.

OCDE (2008a), Politiques de soutien des biocarburants: une évaluation économique, OCDE, Paris.

OCDE (2008b), La performance environnementale de l'agriculture dans les pays de l'OCDE depuis 1990, OCDE, Paris.

OCDE (2008c), Perspectives agricoles de l'OCDE et de la FAO, 2008-2017, OCDE, Paris, FAO, Rome.

Porter, M. E. (1990), The competitive Advantage of Nations, Free Press.

Roberfroid, M. B. (2002), 'Global view on functional foods: European perspectives', British Journal of Nutrition 88, 2: 133-138.

Rodriguez-Pose, A et R. Crescenzi (2005) "R7D Spillovers, Innovation Systems and the Genesis of Regional Growth in Europe" Bruges European Economic Research papers, no.5.

Rosenberg N. (1972), Technology and American Economic Growth, White Plains, M.E. Sharpe, New York.

Rosenberg N. (1995), Exploring the black box, Cambridge, Cambridge university Press

Schumpeter, J. A. (1950), Capitalism, Socialism and Democracy, Harper-Collins.

Sibbel, A. (2007), 'The sustainability of functional foods', Social Science \& Medecine 64: 554-561.

Stern, S., M. Porter et J. Furman, (2000) “The Determinants of national innovatitve capacity”, NBER, working paper 7876 .

Teece, D. (1989), 'Inter-organizational Requirement of the Innovation Process', Managerial and Decision Economics, 35:42.

Teece, D. (2008), 'Dosi's Technological paradigms and trajectories: insights for economics and management', Industrial and corporate change 17, 3:5407-12.

Toyne, P., C. Cowell et T. Mech (2004), 'Marketing Agricultural Sustainability', Rural Industries Research and Development Corporation, Australian Government.

USDA (2004), 'Organic Produce, Price Premiums, and Eco-Labelling in U.S. Farmers' Markets', ERS, VGS-301-01.

Waner, J. (2000), 'The Future of Agriculture: An Introduction' in New Generation Cooperatives: Case Study, Illinois Institute for Rural Affairs, Western Illinois University.

Weill, P., B. Schmitt, G. Chesneau, N. Daniel, F. Safraou et P. Legrand (2002), 'Effect of introducing linseed in livestock diet on blood fatty acid composition of consumers of animal products', Annals of Nutrition and Metabolism 46: 182-191.

Weststrate, J. A., G. van Poppel et P.M. Verschuren (2002), 'Functional foods, trends and future', British Journal of Nutrition 88: S233-S235. 


\section{TAD/CA/APM/WP(2008)15/FINAL}

Winter, M. (2003), 'Embeddness, the new food economy and defensive localism', Journal of Rural Studies 19: $23-32$. 